\title{
A ZERO-ONE LAW FOR UNIFORM DIOPHANTINE APPROXIMATION IN EUCLIDEAN NORM
}

\author{
DMITRY KLEINBOCK AND ANURAG RAO
}

\begin{abstract}
We study a norm sensitive Diophantine approximation problem arising from the work of Davenport and Schmidt on the improvement of Dirichlet's theorem. Its supremum norm case was recently considered by the first-named author and Wadleigh [KWa], and here we extend the set-up by replacing the supremum norm with an arbitrary norm. This gives rise to a class of shrinking target problems for one-parameter diagonal flows on the space of lattices, with the targets being neighborhoods of the critical locus of the suitably scaled norm ball. We use methods from geometry of numbers to generalized a result due to Andersen and Duke [AD] on measure zero and uncountability of the set of numbers (in some cases, matrices) for which Minkowski approximation theorem can be improved. The choice of the Euclidean norm on $\mathbb{R}^{2}$ corresponds to studying geodesics on a hyperbolic surface which visit a decreasing family of balls. An application of the dynamical Borel-Cantelli lemma of Maucourant [Mau] produces, given an approximation function $\psi$, a zero-one law for the set of $\alpha \in \mathbb{R}$ such that for all large enough $t$ the inequality $\left(\frac{\alpha q-p}{\psi(t)}\right)^{2}+\left(\frac{q}{t}\right)^{2}<\frac{2}{\sqrt{3}}$ has non-trivial integer solutions.
\end{abstract}

\section{INTRODUCTION}

The theory of approximation of real numbers by rational numbers starts with Dirichlet's Theorem (1842):

$$
\forall \alpha \in \mathbb{R} \quad \forall t>1 \quad \exists q \in \mathbb{N} \text { with }\left\{\begin{array}{c}
\langle q \alpha\rangle \leq 1 / t \\
q<t
\end{array} ;\right.
$$

here and hereafter $\langle x\rangle$ stands for the distance from $x \in \mathbb{R}$ to a nearest integer. See e.g. [Ca1, Theorem I.I] or [S4, Theorem I.1A]. The standard application of (1.1) is the following corollary:

$$
\forall \alpha \in \mathbb{R} \quad \exists \infty \text { many } q \in \mathbb{N} \text { with }\langle q \alpha\rangle<1 / q .
$$

The two statements above show two possible ways to pose Diophantine approximation problems, often (see e.g. [W]) referred to as uniform vs. asymptotic: that is, looking for solvability of inequalities for all large enough values of certain parameters vs. for infinitely many (a distinction between limsup and liminf sets). The rate of approximation given in (1.1) and (1.2) works for all $\alpha$, which serves as a beginning of the metric theory of Diophantine approximation, concerned with understanding sets of $\alpha$ satisfying similar conclusions but with the right hand sides replaced by faster decaying functions of $t$ and $q$ respectively.

Those sets are well studied in the setting of (1.2). Indeed, for a function $\psi: \mathbb{R}_{+} \rightarrow \mathbb{R}_{+}$ one considers

$$
W(\psi):=\{\alpha \in \mathbb{R}: \exists \infty \text { many } q \in \mathbb{N} \text { with }\langle q \alpha\rangle<\psi(q)\},
$$

the set of $\psi$-approximable real numbers. With the notation $\psi_{k}(t):=1 / t^{k},(1.2)$ asserts that $W\left(\psi_{1}\right)=\mathbb{R}$; moreover a theorem of Hurwitz (see $[\mathrm{S} 4,1.2 \mathrm{~F}]$ ) says that $W\left(c \psi_{1}\right)=\mathbb{R}$ for all $c \geq 1 / \sqrt{5}$. Numbers which do not belong to $W\left(c \psi_{1}\right)$ for some $c>0$ are called badly

Date: July 23, 2020.

Research supported by NSF grants DMS-1600814 and DMS-1900560. 
approximable; we shall denote the set of those numbers by BA. If $\psi$ is non-increasing, Khintchine's Theorem gives the criterion for the Lebesgue measure of $W(\psi)$ to be zero or full - namely, the convergence/divergence of the series $\sum_{k} \psi(k)$.

Let us now briefly describe what is known in the setting of (1.1). Following [KWa], for $\psi$ as above say that $\alpha$ is $\psi$-Dirichlet if for all large enough $t$

$$
\text { there exists } q \in \mathbb{N} \text { with }\left\{\begin{array}{cl}
\langle q \alpha\rangle & <\psi(t) \\
q & <t
\end{array}\right. \text {. }
$$

Let us denote the set of $\psi$-Dirichlet numbers by $D_{\infty}(\psi)$ (the role of the subscript $\infty$ will be clarified below). It is immediate ${ }^{1}$ from $(1.1)$ that $D_{\infty}\left(\psi_{1}\right)=\mathbb{R}$. Also let us say that $\alpha$ is Dirichlet-improvable (see e.g. [EW, Definition 5.8]) if it belongs to $D_{\infty}\left(c \psi_{1}\right)$ for some $c<1$. Denote by

$$
\widehat{D}_{\infty}:=\bigcup_{c<1} D_{\infty}\left(c \psi_{1}\right)
$$

the set of Dirichlet-improvable numbers. Morimoto ([Mo], see also [DS1, Theorem 1]) was the first to observe that the set $\widehat{D}_{\infty}$ coincides with $\mathbb{Q} \cup \mathbf{B A}$, and in particular has Lebesgue measure zero and is thick, that is, intersects any non-empty open subset of $\mathbb{R}$ in a set of full Hausdorff dimension. The latter property, originally established by Jarník [J], was upgraded by Schmidt [S1] to being a winning set, and then further strengthened by McMullen [Mc] to absolute winning; see Remark 3.5 for more detail.

Further progress in the study of the sets $D_{\infty}(\psi)$ was made in a recent paper [KWa] by the first named author and Wadleigh. Namely, the following was proved:

Theorem 1.1 ([KWa], Theorems 1.7 and 1.8). Let $\psi$ be a non-increasing function such that $t \psi(t)<1$ for all sufficiently large $t$. Then

(a) $D_{\infty}(\psi)^{c} \neq \varnothing$;

(b) if, in addition, the function $t \mapsto t \psi(t)$ is non-decreasing, then the Lebesgue measure of $D_{\infty}(\psi)$ (resp. of $\left.D_{\infty}(\psi)^{c}\right)$ is zero if

$$
\sum_{k}-\log (1-k \psi(k))\left(\frac{1}{k}-\psi(k)\right)=\infty \quad(\text { resp. }<\infty) .
$$

The above theorem was proved via a tight description of elements of $D_{\infty}(\psi)$ in terms of their continued fraction expansion. An alternative description can be easily provided via a reduction of the problem to dynamics on the space of lattices in $\mathbb{R}^{2}$. Indeed, let $u_{\alpha}:=\left[\begin{array}{cc}1 & \alpha \\ 0 & 1\end{array}\right]$, and consider

$$
\Lambda_{\alpha}:=u_{\alpha} \mathbb{Z}^{2}=\left\{\left(\begin{array}{c}
\alpha q-p \\
q
\end{array}\right): p, q \in \mathbb{Z}\right\} .
$$

Denote by $B(r)$ the open ball in $\mathbb{R}^{2}$ of radius $r$ centered at 0 with respect to the supremum norm on $\mathbb{R}^{2}$. Then it is easy to see that $\alpha \in D_{\infty}(\psi)$ if and only if for all large enough $t$ the lattice $\Lambda_{\alpha}$ has a nonzero vector inside the rectangular box $\left[\begin{array}{cc}\psi(t) & 0 \\ 0 & t\end{array}\right] B(1)$.

While the use of supremum norm arises naturally from the problem considered by Dirichlet, it seems natural to state similar problems for an arbitrary norm $\nu$, thereby replacing balls $B(r)$ with

$$
B_{\nu}(r):=\left\{\mathbf{x} \in \mathbb{R}^{2}: \nu(\mathbf{x})<r\right\},
$$

\footnotetext{
${ }^{1}$ More precisely, if $\alpha \notin \mathbb{Q}$ (resp. if $\alpha \in \mathbb{Q}$ ), the system (1.3) with $\psi=\psi_{1}$ is solvable for all $t>1$ (resp. for all sufficiently large $t$ ).
} 
open balls centered at 0 with respect to the norm $\nu$. A recent article [AD] by Andersen and Duke provides evidence that this norm sensitive approximation problem was studied by Hermite only a few years after the work of Dirichlet, and later on by Minkowski.

Keeping up with the notation in [AD], we define for each norm $\nu$ a critical value

$$
\Delta_{\nu}:=\text { the smallest co-volume over all lattices intersecting } B_{\nu}(1) \text { trivially. }
$$

Much is known about these constants and the set of lattices that attain this lower bound. For example, $\Delta_{\nu}$ is computed as the minimal area of a parallelogram with one vertex at the origin and the other three on the boundary of $B_{\nu}(1)$.

This critical value is used in our generalization of $D_{\infty}(\psi)$. Namely, let us say that $\alpha$ is $(\psi, \nu)$-Dirichlet, or $\alpha \in D_{\nu}(\psi)$, if

$$
\Lambda_{\alpha} \cap\left[\begin{array}{cc}
\psi(t) & 0 \\
0 & t
\end{array}\right] B_{\nu}\left(\frac{1}{\sqrt{\Delta_{\nu}}}\right) \neq\{0\} \text { whenever } t>0 \text { is large enough. }
$$

Note, this definition is consistent with what we had before since the critical value for the supremum norm is 1 . Furthermore, it is easy to see that the function $\psi_{1}$ again plays the role of a critical parameter: if $c>1$, then $D_{\nu}\left(c \psi_{1}\right)=\mathbb{R}$.

We will always assume $\psi$ to be non-decreasing and continuous. Note that the case $\nu=\|\cdot\|_{\infty}$ has an extra feature: if (1.6) is true for all large enough $t \in \mathbb{N}$, then the same is true for all large enough $t>0$. This makes it possible to reduce the problem to continuous functions $\psi$. This argument does not apply to the set-up of arbitrary norms $\nu$. However, for the most part the scope of our paper will allow us to only deal with the continuous case, see Remark 1.7.

When $\nu(\mathbf{x})=\|\mathbf{x}\|_{p}$, the $\ell^{p}$ norm, we shall denote $B_{\nu}(r)$ by $B_{p}(r), D_{\nu}(\psi)$ by $D_{p}(\psi)$ and $\Delta_{\nu}$ by $\Delta_{p}$; the set-up discussed in (1.3) corresponds to $p=\infty$. In the case of the Euclidean norm, which will be the main topic of this paper, $D_{2}(\psi)$ is the set of $\alpha \in \mathbb{R}$ for which the inequality

$$
\left(\frac{\langle\alpha q\rangle}{\psi(t)}\right)^{2}+\left(\frac{q}{t}\right)^{2}<\frac{2}{\sqrt{3}}
$$

is solvable in $q \in \mathbb{N}$ for all large enough $t$. (Note that $\Delta_{2}=\sqrt{3} / 2$.)

In the paper $[\mathrm{AD}]$, Andersen and Duke obtained several results under the additional assumption that $\nu$ is strongly symmetric, that is satisfies

$$
\nu\left(\left(x_{1}, x_{2}\right)\right)=\nu\left(\left(\left|x_{1}\right|,\left|x_{2}\right|\right)\right) \text { for all }\left(x_{1}, x_{2}\right) \in \mathbb{R}^{2} .
$$

In particular, they considered a generalization of the set $\widehat{D}_{\infty}$ :

$$
\widehat{D}_{\nu}:=\bigcup_{c<1} D_{\nu}\left(c \psi_{1}\right)
$$

which they referred to as 'the set of numbers for which Minkowski's approximation theorem can be improved', and proved

Theorem 1.2 ([AD], Theorem 1.1). For any strongly symmetric norm $\nu$ on $\mathbb{R}^{2}$, the set $\widehat{D}_{\nu}$ (a) has Lebesgue measure zero, and (b) is uncountable.

In the present paper we would like to take an arbitrary norm $\nu$ on $\mathbb{R}^{2}$ and consider the following

\section{Questions.}

(i) Will part (a) of the above theorem hold in that generality?

(ii) Will part (b) hold, and can one strengthen its conclusion by showing that the set $\widehat{D}_{\nu}$ is thick? winning? absolute winning?

(iii) Is it true that $D_{\nu}\left(\psi_{1}\right)=\mathbb{R}$ ? 
(iv) It is true that $D_{\nu}(\psi)^{c} \neq \varnothing$ whenever $\psi(t)<\psi_{1}(t)$ for all sufficiently large $t$ ?

(v) Perhaps under some additional condition such as the monotonicity of the function $t \mapsto t \psi(t)$, can one find a criterion for the Lebesgue measure of $D_{\nu}(\psi)$ to be zero or full?

We answer Question (i) affirmatively in the very general set-up of systems of $m$ linear forms in $n$ variables, where $m, n \in \mathbb{N}$ are arbitrary (Theorem 3.1). With regards to Question (ii), strengthening Theorem 1.2(b), we prove

Theorem 1.3. For any norm $\nu$ on $\mathbb{R}^{2}$, the set $\widehat{D}_{\nu}$ is absolute winning.

We also obtain some partial results in the higher-dimensional case; in particular, a modification of the absolute winning property, namely hyperplane absolute winning (HAW) introduced in [BFKRW], will be shown to hold for a multi-dimensional analogue of the set $\widehat{D}_{\nu}$ where $\nu$ is the Euclidean norm on $\mathbb{R}^{m+n}$ (see Theorem 3.7).

For the rest of the questions we restrict our attention to the Euclidean norm on $\mathbb{R}^{2}$. Specifically, we prove the following theorems:

Theorem 1.4. $D_{2}\left(\psi_{1}\right)=\mathbb{R}$; in other words, for any $\alpha \in \mathbb{R}$ the inequality

$$
\langle\alpha q\rangle<\frac{1}{t} \sqrt{\frac{2}{\sqrt{3}}-\left(\frac{q}{t}\right)^{2}}
$$

is solvable for all large enough $t>0$.

Theorem 1.5. Let $\psi$ be a non-increasing continuous function such that

$$
\psi(t)<\psi_{1}(t)=\frac{1}{t} \quad \text { for all sufficiently large } t .
$$

Then $D_{2}(\psi)^{c} \neq \varnothing$.

Theorem 1.6. Let $\psi$ be as in Theorem 1.5, and assume, in addition, that

$$
\text { the function } t \mapsto t \psi(t) \text { is non-decreasing. }
$$

Then the Lebesgue measure of $D_{2}(\psi)$ (resp. of $\left.D_{2}(\psi)^{c}\right)$ is zero whenever

$$
\sum_{k}\left(\psi_{1}(k)-\psi(k)\right)=\infty \quad(\text { resp. }<\infty) .
$$

Note the difference between (1.10) and (1.4): the latter can be written as

$$
\sum_{k}\left(\psi_{1}(k)-\psi(k)\right) \log \left(\frac{1}{k\left(\psi_{1}(k)-\psi(k)\right)}\right)=\infty \quad(\text { resp. }<\infty) ;
$$

that is, compared with (1.10), has an extra logarithmic term. We have the following examples $^{2}$ demonstrating condition (1.10):

- if $\psi(t)=\frac{1}{t}-\frac{1}{t^{k+1}}$, then $D_{2}(\psi)$ has full measure when $k>0$;

- if $\psi(t)=\frac{1}{t}-\frac{1}{t(\log t)^{k}}$, then $D_{2}(\psi)$ is null for $k \leq 1$ and conull for $k>1$.

Remark 1.7. One can notice that condition (1.9) of Theorem 1.6, together with the assumption that $\psi$ is non-increasing, forces $\psi$ to be continuous. On the other hand, Theorem 1.5 would clearly hold for discontinuous functions as long as (1.8) is replaced by

$$
\inf _{t_{0}<t<t_{1}}\left(\psi_{1}(t)-\psi(t)\right)>0 \text { for all sufficiently large } t_{0} \text { and all } t_{1}>t_{0}
$$

\footnotetext{
${ }^{2}$ These functions $\psi$ are only decreasing for large enough values of $t$ - but clearly only the eventual behavior of $\psi$ is relevant to the problem.
} 
This article is structured as follows. In $\S 2$ we generalize the problems described above to the set-up of systems of $m$ linear forms in $n$ variables, and describe the connection with diagonal flows on the space of lattices. In this generality, i.e. for arbitrary $m$ and $n$, in $\S 3$ we address Questions (i) and (ii) from the above list. The first one is answered for an arbitrary norm in Theorem 3.1. For the second one a sufficient condition for the HAW property of the higher-dimensional analogue of the set (1.7) is deduced from a recent work by An, Guan and the first-named author [AGK]. We use that condition to answer Question (ii) for the Euclidean norm on $\mathbb{R}^{m+n}$ and for arbitrary norm on $\mathbb{R}^{2}$. Theorems 1.4 and 1.5 are proved in $\S 4$ by a geometric argument dealing with geodesics in the upper-half plane. In $\S 6$ we show how to deduce Theorem 1.6 from a corresponding dynamical zero-one law for geodesic flows on finite volume hyperbolic surfaces due to Maucourant [Mau], which is discussed in detail in $\S 5$.

Acknowledgements. The authors would like to thank Jinpeng An, Nikolay Moshchevitin, Lam Pham, Srini Sathiamurthy, Nick Wadleigh and Shucheng Yu for helpful discussions, and the anonymous reviewers for a careful reading of the paper which has led to multiple improvements.

\section{Systems of LineAR FORMS AND REDUCTION TO DYNAMiCS}

In this section we generalize the notion of $(\psi, \nu)$-Dirichlet real numbers to the set-up of systems of linear forms. Fix positive integers $m, n$, put $d=m+n$, and denote by $M_{m, n}$ the space of $m \times n$ matrices with real entries, interpreted as systems of $m$ linear forms in $n$ variables, $\mathbf{x} \mapsto A \mathbf{x}$. Let $\nu$ be an arbitrary norm on $\mathbb{R}^{d}$, and let $\psi$ be a non-negative function defined on an interval $\left[t_{0}, \infty\right)$ for some $t_{0} \geq 1$. Generalizing (1.6), let us say that $M_{m, n}$ is $(\psi, \nu)$-Dirichlet, and write $A \in D_{\nu}(\psi)$, if for every sufficiently large $t>0$ one can find $\mathbf{q} \in \mathbb{Z}^{n} \backslash\{0\}$ and $\mathbf{p} \in \mathbb{Z}^{m}$ such that the vector $\left(\begin{array}{c}A \mathbf{q}-\mathbf{p} \\ \mathbf{q}\end{array}\right)$ is inside the "generalized ellipsoid" $\left[\begin{array}{cc}\psi(t) I_{m} & 0 \\ 0 & t I_{n}\end{array}\right] B_{\nu}\left(\frac{1}{\Delta_{\nu} 1 / d}\right)$, where $\Delta_{\nu}$ is as in (1.5). Here $I_{k}$ stands for the $k \times k$ identity matrix, and, as before, we use notation

$$
B_{\nu}(r):=\left\{\mathbf{x} \in \mathbb{R}^{d}: \nu(\mathbf{x})<r\right\} .
$$

When $\nu=\|\cdot\|_{\infty}$ is the supremum norm on $\mathbb{R}^{d}$, we recover the standard set-up of uniform simultaneous Diophantine approximation: indeed, in that case the condition $A \in D_{\nu}(\psi)$ is equivalent to the system ${ }^{3}$

$$
\left\{\begin{aligned}
\|A \mathbf{q}-\mathbf{p}\|_{\infty} & <\psi(t) \\
\|\mathbf{q}\|_{\infty} & <t
\end{aligned}\right.
$$

having a nonzero solution $(\mathbf{p}, \mathbf{q})$ for all large enough $t$.

Let us now restate the $(\psi, \nu)$-Dirichlet property in the language of dynamics on the space $X=\mathrm{SL}_{d}(\mathbb{R}) / \mathrm{SL}_{d}(\mathbb{Z})$ of unimodular lattices in $\mathbb{R}^{d}$. Define

$$
u_{A}:=\left[\begin{array}{cc}
I_{m} & A \\
0 & I_{n}
\end{array}\right]
$$

and

$$
\Lambda_{A}:=\left\{\left(\begin{array}{c}
A \mathbf{q}-\mathbf{p} \\
\mathbf{q}
\end{array}\right): \mathbf{p} \in \mathbb{Z}^{m}, \mathbf{q} \in \mathbb{Z}^{n}\right\}=u_{A} \mathbb{Z}^{d} \in X
$$

\footnotetext{
${ }^{3}$ This definition differs slightly from the one used in [KWa, §4] and [CGGMS, Definition 2.2], where the system $\left\{\begin{aligned}\|A \mathbf{q}-\mathbf{p}\|_{\infty}^{m} & <\psi(t) \\ \|\mathbf{q}\|_{\infty}^{n} & <t\end{aligned}\right.$ was used in place of $(2.1)$.
} 
then $A \in D_{\nu}(\psi)$ if and only if

$$
\Lambda_{A} \cap\left[\begin{array}{cc}
\psi(t) I_{m} & 0 \\
0 & t I_{n}
\end{array}\right] B_{\nu}\left(\frac{1}{\Delta_{\nu}^{1 / d}}\right) \neq\{0\}
$$

whenever $t>0$ is large enough. Note that the determinant of $\left[\begin{array}{cc}\psi(t) I_{m} & 0 \\ 0 & t I_{n}\end{array}\right]$ is equal to $\psi(t)^{m} t^{n}$; thus, to reduce the problem to the $\mathrm{SL}_{d}(\mathbb{R})$-action on $X$, one can introduce the matrix

$$
b_{t}:=\left[\begin{array}{cc}
\left(\frac{t}{\psi(t)}\right)^{n / d} I_{m} & 0 \\
0 & \left(\frac{\psi(t)}{t}\right)^{m / d} I_{n}
\end{array}\right] \in \mathrm{SL}_{d}(\mathbb{R}) .
$$

Then (2.2) becomes equivalent to

$$
b_{t} \Lambda_{A} \notin \mathcal{K}_{\nu}\left(t^{n / d} \psi(t)^{m / d}\right)
$$

where

$$
\mathcal{K}_{\nu}(r):=\left\{\Lambda \in X: \Lambda \cap B_{\nu}\left(\frac{r}{\Delta_{\nu}{ }^{1 / d}}\right)=\{0\}\right\} .
$$

Note that for any norm $\nu$, any $r>0$ and in any dimension the sets $\mathcal{K}_{\nu}(r)$ are compact in view of Mahler's Compactness Criterion [Ma1].

The use of $b_{t}$ as in (2.3) has two obvious disadvantages: it is not a group parametrization, and its definition depends on the choice of the function $\psi$. It is much more natural to use a group parametrization:

$$
F:=\left\{a_{s}: s \in \mathbb{R}\right\}, \quad \text { where } a_{s}:=\left[\begin{array}{cc}
e^{s / m} I_{m} & 0 \\
0 & e^{-s / n} I_{n}
\end{array}\right] .
$$

This can be achieved by the change of variables

$$
s:=\frac{m n}{d} \ln \left(\frac{t}{\psi(t)}\right),
$$

and then, using the monotonicity and continuity ${ }^{4}$ of $\psi$, to define a function $r:\left[s_{0}, \infty\right) \rightarrow$ $\mathbb{R}_{+}$, where $s_{0}:=\frac{m n}{d} \ln \left(\frac{t_{0}}{\psi\left(t_{0}\right)}\right)$, by the equation

$$
r\left(\frac{m n}{d} \ln \left(\frac{t}{\psi(t)}\right)\right)=t^{n / d} \psi(t)^{m / d} .
$$

The passage from $\psi$ to $r$ and back is usually referred to as the Dani correspondence. (See [KWa, Proposition 4.5] where it is written down for the supremum norm.) We have arrived at the following

Proposition 2.1. Let $\nu$ be an arbitrary norm on $\mathbb{R}^{d}$, let $\psi$ be a non-increasing continuous function, and let $r(\cdot)$ be the unique function related to $\psi$ via (2.6). Then $A \in D_{\nu}(\psi)$ if and only if

$$
a_{s} \Lambda_{A} \notin \mathcal{K}_{\nu}(r(s)) \text { whenever } s \text { is large enough. }
$$

Observe that when $\psi(t)=c \psi_{n / m}(t)=c t^{-n / m}$, one has $t^{n / d} \psi(t)^{m / d} \equiv c^{m / d}$; in other words, under the Dani correspondence

$$
\psi=c \psi_{n / m} \text { corresponds to the constant function } r(s) \equiv c^{m / d} \text {. }
$$

\footnotetext{
${ }^{4}$ The continuity of $\psi$ is needed to uniquely define $r$ in terms of $\psi$ via (2.5). As was noted in the introduction, $\psi$ can be assumed to be continuous in the supremum norm case. To deal with arbitrary norms, specifically the Euclidean norm, the continuity assumption needs to be added. As mentioned in Remark 1.7, the scope of Theorems 1.5 and 1.6 allows one to do this without loss of generality.
} 
By definition of $\Delta_{\nu}, \mathcal{K}_{\nu}(r)=\varnothing$ for $r>1$, which immediately implies that

$$
D_{\nu}\left(c \psi_{n / m}\right)=M_{m, n} \quad \text { whenever } c>1 \text {. }
$$

Note that when $\nu=\|\cdot\|_{\infty}$, the critical value $\Delta_{\infty}$ is equal to 1 in any dimension, and (2.9) corresponds to the classical Dirichlet's Theorem for simultaneous approximation.

Note also that when $r<1, \mathcal{K}_{\nu}(r)$ is a non-empty, compact set containing an open neighborhood of

$$
\mathcal{K}_{\nu}(1)=\bigcap_{r<1} \mathcal{K}_{\nu}(r)
$$

The latter set, called the critical locus corresponding to the norm $\nu$, plays an important role for the problems we are considering; elements of this set are called critical lattices.

Another way of defining the set $\mathcal{K}_{\nu}(1)$ is through the following function on $X$ :

$$
\delta_{\nu}(\Lambda):=\Delta_{\nu}^{1 / d} \inf _{\mathbf{x} \in \Lambda \backslash\{0\}} \nu(\mathbf{x}),
$$

that is, $\delta_{\nu}(\Lambda)$ is the suitably normalized length of a shortest nonzero vector of $\Lambda$. Clearly $\delta_{\nu}$ is continuous, and we have the equality $\mathcal{K}_{\nu}(r)=\delta_{\nu}^{-1}([r, 1])$; in particular, the critical locus $\mathcal{K}_{\nu}(1)=\delta_{\nu}^{-1}(1)$ consists of all lattices maximizing $\delta_{\nu}$, the value of the maximum being equal to 1 due to our normalization.

When $\nu=\|\cdot\|_{\infty}$ is the supremum norm, the structure of its critical locus $\mathcal{K}_{\infty}(1)$ is described by the Hajós-Minkowski Theorem (see [Ca2, §IX.1.3] and also [F, §3.3] for details of the proof). In particular for $d=2$ one has

$$
\mathcal{K}_{\infty}(1)=\left\{\left[\begin{array}{ll}
1 & \alpha \\
0 & 1
\end{array}\right] \mathbb{Z}^{2}: \alpha \in \mathbb{R}\right\} \bigcup\left\{\left[\begin{array}{ll}
1 & 0 \\
\alpha & 1
\end{array}\right] \mathbb{Z}^{2}: \alpha \in \mathbb{R}\right\}
$$

Something can also be said for the case of the Euclidean norm on $\mathbb{R}^{d}$ for arbitrary $d$ (see Theorem 3.7 below). In general, however, each norm comes with its own peculiarities, with difficulty increasing with dimension. In two dimensions an extensive theoretical study of critical loci appears in the papers [Ma2, Ma3] of Mahler. For example, when $d=2$ and $\nu$ a norm with polygonal unit ball or an $\ell^{p}$ norm, the critical locus $\mathcal{K}_{\nu}(1)$ is finite [Ma4, GGM]. See also [KRS] for examples of critical loci of more complicated nature, e.g. of fractional Hausdorff dimension. In higher dimensions one can find in [Ca2, Chapter V] many useful necessary conditions for a lattice to be critical.

Using (2.8) and Proposition 2.1 we can immediately derive a dynamical description for the higher-dimensional analogue of the set (1.7), that is, the set

$$
\widehat{D}_{\nu}:=\bigcup_{c<1} D_{\nu}\left(c \psi_{n / m}\right)
$$

of $\nu$-Dirichlet-improvable systems of linear forms $A \in M_{m, n}$ :

Proposition 2.2. $A \in \widehat{D}_{\nu}$ if and only if there exists an open neighborhood $U$ of $\mathcal{K}_{\nu}(1)$ such that $a_{s} \Lambda_{A} \notin U$ for large enough s.

\section{3. $\widehat{D}_{\nu}$ IS A WINNING SET OF MEASURE ZERO}

One implication of the correspondence described in the preceding section is an affirmative action to Question (i) from the introduction:

Theorem 3.1. For any norm $\nu$ on $\mathbb{R}^{d}$, the set $\widehat{D}_{\nu}$ has Lebesgue measure zero.

In view of Proposition 2.2, it is clear that the above theorem immediately follows from 
Proposition 3.2. For Lebesgue-almost every $A \in M_{m, n}$ the trajectory

$$
\left\{a_{s} \Lambda_{A}: s>0\right\}
$$

is dense in $X$.

For the case $\min (m, n)=1$ the proof of Proposition 3.2, which capitalizes on [DS2] and is based on geometry of numbers, can be found in [S3]. In [DS2] a slightly weaker statement was used to establish Theorem 3.1 for $\nu=\|\cdot\|_{\infty}$. It is not clear if the argument of [S3] extends to arbitrary $m, n$. However, as first observed by Dani, the above proposition can be easily derived from the ergodicity of the $a_{s}$-action on $X$.

Proof of Proposition 3.2. The argument is fairly standard. We need to prove that for Lebesgue-a.e. $A \in M_{m, n}$ the trajectory $\left\{a_{s} \Lambda_{A}: s>0\right\}$ is dense in $X$. It is easy to see that elements $g \in \mathrm{SL}_{d}(\mathbb{R})$ of the form

$$
g=\left[\begin{array}{cc}
I_{m} & 0 \\
C & I_{n}
\end{array}\right]\left[\begin{array}{cc}
B & 0 \\
0 & D
\end{array}\right] u_{A}
$$

where $A \in M_{m, n}, C \in M_{n, m}, B \in M_{m, m}, D \in M_{n, n}$ with $\operatorname{det}(B) \operatorname{det}(D)=1$, form an open dense subset of $\mathrm{SL}_{d}(\mathbb{R})$ of full Haar measure. That is, $\mathrm{SL}_{d}(\mathbb{R})$ is locally a direct product of

$$
H_{-}:=\left\{\left[\begin{array}{cc}
I_{m} & 0 \\
C & I_{n}
\end{array}\right]: C \in M_{n, m}\right\}, H:=\left\{u_{A}: A \in M_{m, n}\right\}
$$

(those are the contracting and expanding horospherical subgroups ${ }^{5}$ relative to $\left\{a_{s}: s>0\right\}$ ), and the centralizer

$$
Z=\left\{\left[\begin{array}{cc}
B & 0 \\
0 & D
\end{array}\right]: B \in M_{m, m}, D \in M_{n, n}\right\}
$$

of $a_{s}$. On the other hand, the ergodicity of the $a_{s}$-action on $X$ (Moore's Ergodicity Theorem) implies that for Haar-a.e. $g \in \mathrm{SL}_{d}(\mathbb{R})$ the trajectory $\left\{a_{s} g \mathbb{Z}^{d}: s>0\right\}$ is dense in $X$. Now one can write

$$
a_{s} g \mathbb{Z}^{d}=\left[\begin{array}{cc}
I_{m} & 0 \\
e^{-\frac{d}{m n} s} C & I_{n}
\end{array}\right]\left[\begin{array}{cc}
B & 0 \\
0 & D
\end{array}\right] a_{s} \Lambda_{A},
$$

and, since $\left[\begin{array}{cc}I_{m} & 0 \\ e^{-\frac{d}{m n} s} C & I_{n}\end{array}\right]$ tends to $I_{d}$ as $s \rightarrow \infty$, conclude that, for $g$ of the form (3.2), $\left\{a_{s} g \mathbb{Z}^{d}: s>0\right\}$ is dense in $X$ if and only if so is $\left\{a_{s} \Lambda_{A}: s>0\right\}$. The claim then follows from Fubini's Theorem and the local product structure of Haar measure on $\mathrm{SL}_{d}(\mathbb{R})$.

Let us now address Question (ii) from the introduction in the bigger generality of systems of linear forms, that is, construct sufficiently many $\nu$-Dirichlet-improvable $A \in M_{m, n}$. In view of Proposition 2.2 the problem can be restated as follows: find sufficiently many $A \in M_{m, n}$ such that the set of limit points of the trajectory (3.1) is disjoint from $\mathcal{K}_{\nu}(1)$. This circle of problems has a rich history, see $[\mathrm{Kl}, \mathrm{AGK}]$ and references therein. In order to use some results from the aforementioned papers we need to introduce some more terminology.

Definition 3.3. Let $G$ be a Lie group, $\Gamma$ a discrete subgroup, $Z$ a $\mathcal{C}^{1}$ submanifold of $G / \Gamma$, and let $F$ and $H$ be two closed subgroups of $G$. We use $T_{x}(\cdot)$ to denote the tangent space to a manifold at a point $x$, and $\operatorname{Lie}(\cdot)$ to denote the Lie algebra of a group, i.e. the tangent space at the identity element of the group. We will say that $Z$ is $(F, H)$-transversal at $x \in Z$ if the following holds:

\footnotetext{
${ }^{5}$ A subgroup $H$ of a Lie group $G$ is said to be expanding horospherical relative to a one-parameter semigroup $\left\{a_{s}: s>0\right\} \subset G$ if its Lie algebra is a direct sum of generalized eigenspaces of $\operatorname{Ad}\left(a_{1}\right)$ with eigenvalues of absolute values bigger than 1; the contracting horospherical subgroup is defined similarly.
} 
(i) $T_{x}(F x) \not \subset T_{x} Z$;

(ii) $T_{x}(H x) \not \subset T_{x} Z \oplus T_{x}(F x)$.

We will say that $Z$ is $(F, H)$-transversal if it is $(F, H)$-transversal at its every point. This is a simplified version of the terminology introduced in [Kl, §4]. Note that in a special case when $Z$ is an orbit of a Lie subgroup $L$ of $G$, the above conditions (i), (ii) can be easily restated as

$$
\operatorname{Lie}(F) \not \subset \operatorname{Lie}(L)
$$

and

$$
\operatorname{Lie}(H) \not \subset \operatorname{Lie}(L) \oplus \operatorname{Lie}(F)
$$

respectively.

The following was proved in [Kl] for arbitrary $G$ and $\Gamma$, see [Kl, Corollary 4.3.2]: if $F=\left\{a_{s}: s \in \mathbb{R}\right\}$ is a non-quasiunipotent ${ }^{6}$ one-parameter subgroup of $G$, and $H$ is the expanding horospherical subgroup relative to $\left\{a_{s}: s>0\right\}$, then for any $\mathcal{C}^{1}$ compact $(F, H)$-transversal submanifold $Z$ of $G / \Gamma$ and any $x \in X$, the set

$$
\left\{h \in H: \overline{\left\{a_{s} h x: s \geq 0\right\}} \cap Z=\varnothing\right\}
$$

is thick. This has been strengthened in a recent work of An, Guan and the first-named author $[\mathrm{AGK}]$. Namely, they introduced a notion of maximally expanding horospherical subgroup of $G$ relative to $\left\{a_{s}: s>0\right\}$, which, in the special case of $\operatorname{Ad}\left(a_{s}\right)$ being diagonalizable over $\mathbb{R}$, is defined as a subgroup of $G$ whose Lie algebra is the sum of (real) eigenspaces corresponding to eigenvalues of $\operatorname{Ad}\left(a_{1}\right)$ with maximum absolute value. It is always contained in the expanding horospherical subgroup, and in the special case of $F$ as in (2.4) clearly coincides with $H$ as in (3.3).

The next theorem is a special case of [AGK, Theorem 2.8]:

Theorem 3.4. Let $G$ be a Lie group, $\Gamma$ a discrete subgroup, $F=\left\{a_{s}: s \in \mathbb{R}\right\}$ a oneparameter subgroup which is Ad-diagonalizable over $\mathbb{R}, H$ the maximally expanding horospherical subgroup of $G$ relative to $\left\{a_{s}: s>0\right\}$, and $Z$ a $\mathcal{C}^{1}(F, H)$-transversal submanifold of $G / \Gamma$. Then for any $x \in G / \Gamma$, the set (3.6) is $H A W$.

Remark 3.5. Hyperplane absolute winning (HAW) property of subsets of Euclidean spaces has been introduced in [BFKRW], and later extended to subsets of smooth manifolds in [KWe]. When the ambient manifold is one-dimensional, this notion coincides with absolute winning as introduced by McMullen [Mc]. We refer the reader to those papers, as well as to [AGK, §2.1], for definitions and more information. The important aspects are that the HAW property is stable under countable intersections and implies winning in the sense of Schmidt [S1], which, in its turn, implies thickness.

Applying Theorem 3.4 to $x=\mathbb{Z}^{d} \in X=\mathrm{SL}_{d}(\mathbb{R}) / \mathrm{SL}_{d}(\mathbb{Z})$ and using Proposition 2.2, we immediately obtain

Corollary 3.6. Let $F$ be as in (2.4) and $H$ as in (3.3), and let $\nu$ be a norm on $\mathbb{R}^{d}$. Suppose that

$$
\begin{aligned}
& \text { the critical locus } \mathcal{K}_{\nu}(1) \text { is contained in the union of finitely many } \\
& \qquad \mathcal{C}^{1} \text { compact }(F, H) \text {-transversal submanifolds of } X .
\end{aligned}
$$

Then $\widehat{D}_{\nu} \subset M_{m, n}$ is hyperplane absolute winning.

\footnotetext{
6i.e. $\operatorname{Ad}\left(a_{1}\right)$ has an eigenvalue with absolute value different from 1
} 
Note that condition (3.7) is not satisfied for the supremum norm, simply because the whole orbit $H \mathbb{Z}^{d}$ belongs to $\mathcal{K}_{\infty}(1)$. However the conclusion of Corollary 3.6 still holds for $\nu=\|\cdot\|_{\infty}$ due to a theorem of Davenport and Schmidt: it is proved in [DS2] that $\widehat{D}_{\infty}$ contains the set of badly approximable systems of linear forms. The latter was shown by Schmidt to be winning [S2], and, more recently, Broderick, Fishman and Simmons [BFS] established its HAW property.

We will now consider two special cases. The first is the Euclidean norm in arbitrary dimension. Lattices critical with respect to the Euclidean norm have been studied as far back as the 17th century in the context of sphere packings, and later in the context of positive definite quadratic forms. See the book of Martinet [Mar] for a detailed account and exhaustive references.

Theorem 3.7. The critical locus $\mathcal{K}_{2}(1) \subset X$ corresponding to the Euclidean norm on $\mathbb{R}^{d}$ is contained in the union of finitely many $\mathrm{SO}(d)$-orbits, and each orbit is an $(F, H)$ transversal submanifold of $X$. Consequently, $\widehat{D}_{2} \subset M_{m, n}$ is $H A W$.

Proof. It follows form the work of Korkine and Zolotareff ([KZ], see also [Mar, Theorem 3.4.5]) that whenever $\Lambda \in \mathcal{K}_{2}(1)$, any lattice in $X$ sufficiently close to $\Lambda$ is an isometric image of $\Lambda$, that is, a lattice of the form $g \Lambda$ with $g \in \mathrm{SO}(d)$. For the sake of making the paper self-contained we state the lemmas required to prove this and indicate how to use them.

Lemma 3.8 ([Mar], Lemma 3.4.2). Let $\Lambda$ be any lattice in $\mathbb{R}^{d}$. Then there exists a neighborhood $V$ of the identity in $\mathrm{GL}_{d}(\mathbb{R})$ such that for any $g \in V$ the nonzero vectors in $g \Lambda$ of minimal Euclidean norm are images under $g$ of such minimal vectors in $\Lambda$.

Lemma 3.9 ([Mar], Lemma 3.4.4(1)). There is an open neighborhood $W$ of 0 in the vector space $\operatorname{Sym}_{d}(\mathbb{R})$ of real symmetric $d \times d$ matrices such that, for $h \in W$ with $\operatorname{tr}(h) \leq 0$ and for $g \in \mathrm{GL}_{d}(\mathbb{R})$ satisfying $g^{t} g=I_{d}+h$, we have either $g \in \mathrm{O}(d)$ or $\operatorname{det}(g)<1$.

Lemma 3.10 ([Mar], Lemma 3.4.4(2)). Let $C$ be a closed cone in $\operatorname{Sym}_{d}(\mathbb{R})$ consisting of matrices with positive trace:

$$
\operatorname{tr}(h)>0 \text { for every nonzero } h \in C .
$$

Then there exists a neighborhood $W_{C}$ of 0 in $\operatorname{Sym}_{d}(\mathbb{R})$ such that

$$
h \neq 0 \text { and } h \in W_{C} \cap C \Longrightarrow \operatorname{det}\left(I_{d}+h\right)>1 .
$$

Let us now proceed with the proof of Theorem 3.7. Take $\Lambda_{0} \in \mathcal{K}_{2}(1)$, and suppose $g_{k} \in \mathrm{SL}_{d}(\mathbb{R}) \backslash \mathrm{SO}(d)$ are such that $g_{k}$ converges to the identity as $k \rightarrow \infty$ and $g_{k} \Lambda_{0} \in \mathcal{K}_{2}(1)$ for all $k$. Define symmetric matrices $h_{k}$ by setting $g_{k}^{t} g_{k}=I_{d}+h_{k}$; note that $h_{k} \neq 0$ since $g_{k} \notin \mathrm{SO}(d)$. We first claim that each $h_{k}$ belongs to the closed cone

$$
C:=\left\{h \in \operatorname{Sym}_{d}(\mathbb{R}): \mathbf{v}^{t} h \mathbf{v} \geq 0 \text { for all } \mathbf{v} \in \Lambda_{0} \backslash\{0\} \text { with }\|\mathbf{v}\|_{2} \text { minimal }\right\} .
$$

For this, note that for any $\mathbf{v} \in \mathbb{R}^{d}$ we have

$$
\mathbf{v}^{t} h_{k} \mathbf{v}=\mathbf{v}^{t}\left(g_{k}^{t} g_{k}-I_{d}\right) \mathbf{v}=\left\|g_{k} \mathbf{v}\right\|_{2}^{2}-\|\mathbf{v}\|_{2}^{2}
$$

Since both $\Lambda_{0}$ and $g_{k} \Lambda_{0}$ are in $\mathcal{K}_{2}(1)$, for any nonzero $\mathbf{v} \in \Lambda_{0}$ with minimal norm this implies $\left\|g_{k} \mathbf{v}\right\|_{2}^{2}-\|\mathbf{v}\|_{2}^{2} \geq 0$, which proves the claim.

The next claim is that $C$ as in (3.9) satisfies (3.8). Indeed, for any nonzero $h \in C$ consider the suitably scaled matrix $h^{\prime}=c h(c>0)$ which lies in $W \cap C, W$ being the open set in Lemma 3.9. By further decreasing $c$ we can assume that there exists $g \in V$, where $V$ is as in Lemma 3.8, such that $g^{t} g=I_{d}+h^{\prime}$. Since $c>0$, it suffices to prove that $\operatorname{tr}\left(h^{\prime}\right)>0$. 
Assume, on the contrary, that $\operatorname{tr}\left(h^{\prime}\right) \leq 0$. Lemma 3.9 then says that we must have $g \in \mathrm{O}(d)$ or $\operatorname{det}(g)<1$. The fact that $h$ is nonzero precludes the first alternative, and so it follows that $\operatorname{det}(g)<1$. Now consider the unimodular lattice

$$
\Lambda:=\frac{1}{\operatorname{det}(g)^{1 / d}} g \Lambda_{0} .
$$

For any nonzero vector $\mathbf{v} \in \Lambda_{0}$ of minimal length we have that

$$
\left\|\frac{1}{\operatorname{det}(g)^{1 / d}} g \mathbf{v}\right\|_{2}^{2}=\frac{1}{\operatorname{det}(g)^{2 / d}} \mathbf{v}^{t} g^{t} g \mathbf{v}=\frac{1}{\operatorname{det}(g)^{2 / d}}\left(\|\mathbf{v}\|_{2}^{2}+\mathbf{v}^{t} h^{\prime} \mathbf{v}\right)>\|\mathbf{v}\|_{2}^{2}
$$

since $h^{\prime} \in C$ and $\operatorname{det}(g)<1$. Lemma 3.8 then shows that the length of the shortest vector in $\Lambda$ must be greater than that for $\Lambda_{0}$. However the fact that $\Lambda_{0} \in \mathcal{K}_{2}(1)$ actually implies that $\Lambda_{0}$ is a global maximum of the function $\delta_{2}$ defined in $(2.10)$ with $\|\cdot\|=\nu_{2}$, a contradiction.

Thus our claim is proved, and the stage is set for applying Lemma 3.10. Indeed, we have $h_{k} \in C \backslash\{0\}$; since $h_{k} \rightarrow 0$, we can assume that $h_{k} \in W_{C}$ for large enough $k$. Hence $\operatorname{det}\left(I_{d}+h_{k}\right)=\operatorname{det}\left(g_{k}\right)^{2}>1$, contradicting the assumption that $g_{k} \in \mathrm{SL}_{d}(\mathbb{R})$.

This argument, together with compactness of $\mathcal{K}_{2}(1)$, implies that the critical locus $\mathcal{K}_{2}(1)$ is contained in the union of finitely many $\mathrm{SO}(d)$-orbits. Thus it suffices to check the transversality conditions for $Z$ being a single orbit; that is, the validity of (3.4) and (3.5) for $L=\mathrm{SO}(d)$. The latter is straightforward, since $\operatorname{Lie}(L)=\mathfrak{s o}(d)$ consists of skew-symmetric matrices and hence does not contain $\operatorname{Lie}(F)$ for $F$ as in $(2.4)$; likewise, $\operatorname{Lie}(H)$ for $H$ as in (3.3) consists of upper-triangular matrices and hence is not contained in $\mathfrak{s o}(d) \oplus \operatorname{Lie}(F)$.

From now until the end of the paper we restrict our attention to $m=n=1$ and prove Theorems 1.3-1.6. Recall that in this low-dimensional case we are working with $X=G / \Gamma$, where $G=\mathrm{SL}_{2}(\mathbb{R})$ and $\Gamma=\mathrm{SL}_{2}(\mathbb{Z})$, and the subgroups $F, H$ of $G$ are one-parameter of the form

$$
H=\left\{u_{\alpha}: \alpha \in \mathbb{R}\right\}, F=\left\{a_{s}: s \in \mathbb{R}\right\}, \text { where } u_{\alpha}=\left[\begin{array}{cc}
1 & \alpha \\
0 & 1
\end{array}\right], a_{s}=\left[\begin{array}{cc}
e^{s} & 0 \\
0 & e^{-s}
\end{array}\right] .
$$

For any point $x \in X$, the left action of $G$ on $X$ induces a local diffeomorphism $G \rightarrow X$, $g \mapsto g x$. We identify the tangent space $T_{x}(X)$ with $\operatorname{Lie}(G)$ through this map. Thus any subalgebra of $\operatorname{Lie}(G)$ defines a distribution in the tangent bundle $T(X)$. Note that in the case when $Z \subset X$ is a one-dimensional submanifold and with $F, H$ as above, it is easy to check that the transversality conditions of Definition 3.3 are equivalent to the statement that at each $z \in Z$,

$$
T_{z}(Z) \text { is not contained in the distribution generated by } \operatorname{Lie}(P),
$$

where $P:=\left\{\left[\begin{array}{cc}a & b \\ 0 & a^{-1}\end{array}\right]\right\}$ is the group of upper-triangular $2 \times 2$ matrices.

Another case for which we verify (3.7) is for norms in $\mathbb{R}^{2}$ whose unit balls are not parallelograms. Due to the nature of the argument, we have relegated the proof of the following theorem to the Appendix:

Theorem 3.11. If $\nu$ is a norm in $\mathbb{R}^{2}$ whose unit ball is not a parallelogram, the critical locus $\mathcal{K}_{\nu}(1)$ is contained in a one-dimensional $(F, H)$-transversal $\mathcal{C}^{1}$-submanifold of $X$.

Proof of Theorem 1.3 assuming Theorem 3.11. Recall that we are given an arbitrary norm on $\mathbb{R}^{2}$ and need to prove that $\widehat{D}_{\nu} \subset \mathbb{R}$ is absolute winning. The latter notion, as was mentioned in Remark 3.5, is a one-dimensional version of the HAW property. Theorem 
3.11 verifies (3.7) for norms whose unit balls are not parallelograms, thus the conclusion of Theorem 1.3 in this case follows from Theorem 3.4 and Proposition 2.2.

It remains to consider the case $\nu(\mathbf{x})=\lambda\left\|g^{-1} \mathbf{x}\right\|_{\infty}$ for some $g \in \mathrm{SL}_{2}(\mathbb{R})$ and $\lambda \in \mathbb{R}_{>0}$. In this case the critical locus $\mathcal{K}_{\nu}(1)$ of $\nu$ coincides with $g \mathcal{K}_{\infty}(1)$. Recall that, according to $(2.11), \mathcal{K}_{\infty}(1)$ is equal to the union of two compact one-dimensional manifolds, namely

$$
\mathcal{K}_{\infty}(1)=H \mathbb{Z}^{2} \cup\left(\theta H \theta^{-1}\right) \mathbb{Z}^{2}=H \mathbb{Z}^{2} \cup \theta H \mathbb{Z}^{2}, \text { where } \theta=\left[\begin{array}{cc}
0 & -1 \\
1 & 0
\end{array}\right] .
$$

Therefore $\mathcal{K}_{\nu}(1)$ can be written as $Z_{1} \cup Z_{2}$, where

$$
Z_{1}:=g H \mathbb{Z}^{2}=\left(g H g^{-1}\right) g \mathbb{Z}^{2} \text { and } Z_{2}:=g \theta H \mathbb{Z}^{2}=\left(g \theta H(g \theta)^{-1}\right) g \mathbb{Z}^{2},
$$

i.e. it is the union of two closed orbits of the lattice $g \mathbb{Z}^{2}$ by subgroups conjugate to $H$.

Let us start with $Z_{1}$ and consider two cases:

- If $g \mathrm{Hg}^{-1}$ is not contained in $P$, then (3.11) holds for $Z=Z_{1}$, which implies that $Z_{1}$ is $(F, H)$-transversal. Thus it follows from Theorem 3.4 that the set of $\alpha \in \mathbb{R}$ such that

$$
\text { there are no limit points of }\left\{a_{s} u_{\alpha} \mathbb{Z}^{2}: s \geq 0\right\} \text { in } Z_{1}
$$

is absolute winning.

- $g H g^{-1} \subset P$; this happens if and only if $g=a_{s_{0}} h$ for some $s_{0} \in \mathbb{R}$ and $h \in H$; hence $Z_{1}=a_{s_{0}} H \mathbb{Z}^{2}$. Clearly then (3.12) is equivalent to the statement that

there are no limit points of $\left\{a_{s} u_{\alpha} \mathbb{Z}^{2}: s \geq 0\right\}$ in $H \mathbb{Z}^{2}$.

But (3.13) is satisfied for any $\alpha \in \widehat{D}_{\infty}$, again in view of Proposition 2.2 and the description of the critical locus for the supremum norm. Since $\widehat{D}_{\infty}$ is known to be absolute winning (as was mentioned in the introduction, it contains the set BA which was shown to be absolute winning by McMullen [Mc]), it follows that the set of $\alpha \in \mathbb{R}$ satisfying (3.12) is absolute winning in this case as well.

The argument taking care of $Z_{2}$ is identical, with $g$ replaced by $g \theta$. Using the intersection property of absolute winning sets, we conclude that the set

$$
\widehat{D}_{\nu}=\left\{\alpha \in \mathbb{R}: \text { there are no limit points of }\left\{a_{s} u_{\alpha} \mathbb{Z}^{2}: s \geq 0\right\} \text { in } Z_{1} \cup Z_{2}\right\}
$$

is absolute winning.

\section{The tARGEts in The UPPER HALF-PLANE}

For the remaining part of the paper we will only consider the Euclidean norm on $\mathbb{R}^{2}$. To simplify notation from now on we will drop the "Euclidean" subscript 2 whenever it does not cause confusion, that is, denote by $B(r)$ the Euclidean ball in $\mathbb{R}^{2}$ of radius $r$ centered at $0 \in \mathbb{R}^{2}$, and by $\mathcal{K}(r), r \leq 1$, the subsets of the space $X=G / \Gamma$ of unimodular lattices in $\mathbb{R}^{2}$ given by

$$
\mathcal{K}(r)=\{\Lambda \in X: \Lambda \cap B(r / \sqrt{\Delta})=\{0\}\}
$$

where $\Delta=\sqrt{3} / 2$. Then we will have

$$
D(\psi)=\left\{\alpha \in \mathbb{R}: a_{s} \Lambda_{\alpha} \notin \mathcal{K}(r(s)) \text { whenever } s \text { is large enough }\right\} .
$$

Since the norm $\|\cdot\|$ is rotation-invariant, so are the sets (4.1) for any $r$. Furthermore, with the notation $K:=\mathrm{SO}(2)$, we see that the critical locus $\mathcal{K}(1)$ is the $K$-orbit of a single 
lattice, namely the hexagonal lattice inscribed in a disk of radius $1 / \sqrt{\Delta}=\sqrt{2 / \sqrt{3}}$. In other words,

$$
\mathcal{K}(1)=K g_{0} \mathbb{Z}^{2}, \text { where } g_{0}:=\left[\begin{array}{cc}
1 / \sqrt{\Delta} & 1 / 2 \sqrt{\Delta} \\
0 & \sqrt{\Delta}
\end{array}\right] \in G .
$$

See [Ca2, page 32] for a proof.

In view of the rotational invariance of the problem it is natural to move it to the quotient space of $G$ by $K$, that is, to the hyperbolic plane. Let $\mathbb{H}$ denote the half-plane of complex numbers $z=x+i y$ with $y>0$. We identify the tangent space $T \mathbb{H}$ with $\mathbb{H} \times \mathbb{C}$ and give it the Riemannian metric $\frac{d x \otimes d x+d y \otimes d y}{y^{2}}$. $T^{1} \mathbb{H}$ is the set of unit tangents vectors, explicitly given as $\left(x+i y, \xi_{1}+i \xi_{2}\right)$ with $\frac{\xi_{1}^{2}+\xi_{2}^{2}}{y^{2}}=1$. The Möbius action of $G$ on $\mathbb{H}$ is defined as

$$
g z=\left[\begin{array}{ll}
a & b \\
c & d
\end{array}\right] z:=\frac{a z+b}{c z+d}
$$

and is an isometry in this metric. Thus we have an induced left action of $G$ on $T^{1} \mathbb{H}$ given by

$$
g(z, \xi):=\left(\frac{a z+b}{c z+d}, \frac{\xi}{(c z+d)^{2}}\right) .
$$

The action is in fact transitive and (up to the subgroup of index 2) free.

In order to make use of the left $K$-invariance of $\mathcal{K}(r)$, we work with the following right actions $T^{1} \mathbb{H} \curvearrowleft G$ (and also $\mathbb{H} \curvearrowleft G$ ):

$$
(z, \xi) \cdot g:=g^{-1}(z, \xi), \quad z \cdot g:=g^{-1} z .
$$

We use these actions to obtain a bi-equivariant double cover $\phi: G \rightarrow T^{1} \mathbb{H}: \phi(g)=(i, i) \cdot g$. Moreover, $\phi$ descends to a diffeomorphism, which we will also denote by $\phi$, of the left $G$-spaces $X$ and $T^{1} \mathbb{H} / \Gamma$, which is a circle bundle (away from two points) over the manifold $\Sigma:=\mathbb{H} / \Gamma$. With some abuse of notation, let us denote by $\eta$ (resp. $\pi$ ) all the projections to quotients by $\Gamma$ (resp. from tangent bundles to base spaces). We thus have the following commuting diagram:

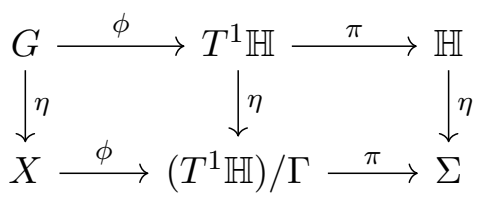

Our goal now is to describe the sets $D(\psi)$ dynamically by restating Proposition 2.1 in the language of hyperbolic geometry. We shall identify the subsets $\mathcal{K}(r)$ of $X$ with their images under $\phi$; their rotation-invariance implies that $\mathcal{K}(r)=\pi^{-1}(\pi(\mathcal{K}(r)))$ for any $r$. Furthermore, let us put $\left(z_{0}, \xi_{0}\right):=\phi\left(g_{0}\right)=(i, i) \cdot g_{0}$, where $g_{0}$ is as in (4.2). Then

$$
z_{0}:=g_{0}^{-1} i=-\frac{1}{2}+i \frac{\sqrt{3}}{2} \in \mathbb{H},
$$

and (4.2) can be used to describe the $\phi$-image of the critical locus $\mathcal{K}(1)$ in $T^{1} \mathbb{H} / \Gamma$ as

$$
\mathcal{K}(1)=\pi^{-1}\left(\eta\left(z_{0}\right)\right)
$$




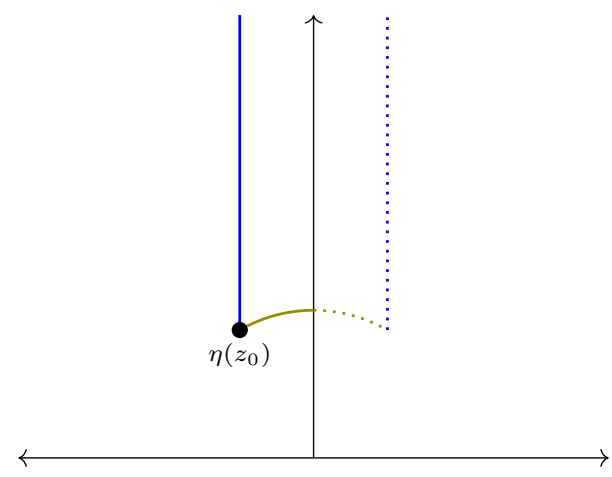

The projection of the critical locus to $\Sigma$

In what follows it will be useful to consider the preimage $\eta^{-1}(\mathcal{K}(r))$ of $\mathcal{K}(r)$ in $T^{1} \mathbb{H}$ as well as in $G$. We will use the notation $\widetilde{\mathcal{K}}(r)$ for both of these sets, context making clear which is in use. The above observations imply that

$$
\pi(\widetilde{\mathcal{K}}(1))=\text { the } \Gamma \text {-orbit of } z_{0} \text { in } \mathbb{H} \text {. }
$$

Now take $\alpha \in \mathbb{R}$ and observe that $\phi$ sends $u_{\alpha}=\left[\begin{array}{ll}1 & \alpha \\ 0 & 1\end{array}\right]$ to $(-\alpha+i, i)$. In other words,

$$
\phi\left(\Lambda_{\alpha}\right)=\eta((-\alpha+i, i))
$$

lies on the closed horocycle on $T^{1} \mathbb{H} / \Gamma$ passing through $\eta(i, i)$. Furthermore, the action of $a_{s}=\left[\begin{array}{cc}e^{s} & 0 \\ 0 & e^{-s}\end{array}\right]$ on $G$ and on $X$ translates into the (negative time direction) geodesic flow on $T^{1} \mathbb{H}$. That is,

$$
\phi\left(a_{s} \Lambda_{\alpha}\right)=\eta\left(\left(-\alpha+e^{-2 s} i, e^{-2 s} i\right)\right) .
$$

We have arrived at the following geometric restatement of Proposition 2.1 for the case of Euclidean norm on $\mathbb{R}^{2}$ :

Proposition 4.1. For any non-increasing continuous $\psi$, let $r(\cdot)$ be the unique function related to $\psi$ via

$$
r\left(\frac{1}{2} \ln \left(\frac{t}{\psi(t)}\right)\right)=\sqrt{t \psi(t)}
$$

which is the $m=n=1$ case of (2.6). Then

$$
\begin{aligned}
\alpha \in D(\psi)^{c} & \Longleftrightarrow a_{s} \Lambda_{\alpha} \in \mathcal{K}(r(s)) \text { for an unbounded set of } s>0 \\
& \Longleftrightarrow-\alpha+e^{-2 s} i \in \pi(\widetilde{\mathcal{K}}(r(s))) \text { for an unbounded set of } s>0 .
\end{aligned}
$$

This enables us to easily answer Questions (iii) and (iv) from the introduction for the case of the Euclidean norm on $\mathbb{R}^{2}$, and to lay a crucial groundwork for our approach to Question (v).

Proof of Theorem 1.4. When $\psi=\psi_{1}, r(s)$ becomes the constant function $r(s) \equiv 1$. Thus, in view of (4.6) and (4.9), $\alpha \in D\left(\psi_{1}\right)^{c}$ if and only if the ray $\left\{-\alpha+e^{-2 s} i: s>0\right\}$ hits the $\Gamma$-orbit of $z_{0}$ for an unbounded set of $s$. However an elementary computation using (4.4) shows that for $\left(\begin{array}{ll}a & b \\ c & d\end{array}\right) \in \Gamma$ one has

$$
\operatorname{Re}\left(\frac{a z_{0}+b}{c z_{0}+d}\right)=\frac{a c+b d-\frac{a d+b c}{2}}{c^{2}-c d+d^{2}} \in \mathbb{Q} .
$$


Therefore $\alpha \in \mathbb{Q}$, which implies that the trajectory (4.7) diverges in $X$, thus cannot return to a compact set infinitely many times.

Proof of Theorem 1.5. Let $\psi$ be any continuous, non-increasing function satisfying (1.8). Then, in view of $(4.8), r(s)$ is strictly less than 1 for all large enough $s$, whence $\pi(\widetilde{\mathcal{K}}(r(s)))$ is a set whose interior contains the $\Gamma$-orbit of $z_{0}$. To show $D(\psi)^{c} \neq \varnothing$ in this case, we use the simple observation that the set of real parts of $\left\{\gamma z_{0}: \gamma \in \Gamma\right\}$ is dense in $\mathbb{R}$. We may thus choose, inductively, a sequence $\left(\gamma_{k}\right) \subset \Gamma$ along with rectangular neighborhoods $U_{k}=A_{k} \times B_{k}$ of $\gamma_{k} z_{0}$ such that $\overline{A_{k+1}} \subset A_{k}$ and $U_{k} \subset \pi\left(\widetilde{\mathcal{K}}\left(r\left(s_{k}\right)\right)\right)$ for every $k$, where $s_{k}$ is defined by $e^{-2 s_{k}}=\operatorname{Im}\left(\gamma_{k} z_{0}\right)$. Then $s_{k} \rightarrow \infty$, and (4.9) shows that any element of $\bigcap A_{n}$ belongs to $D_{2}(\psi)^{c}$. Hence the latter set is non-empty. In fact, a 'Cantor set' type argument will show that $D_{2}(\psi)^{c}$ is uncountable.

The rest of the paper is devoted to proving Theorem 1.6, which, in view of Proposition 4.1 , deals with geodesics in $\mathbb{H} / \Gamma$ visiting a nested sequence of sets $\pi(\mathcal{K}(r))$, which as $r \rightarrow 1$ converge to $\pi(\mathcal{K}(1))=\eta\left(z_{0}\right)$. The goal of the remaining part of this section is to show that the sets $\pi(\mathcal{K}(r))$ with $r$ sufficiently close to 1 can be efficiently approximated by small balls centered at $\eta\left(z_{0}\right)$ :

Proposition 4.2. The exist positive constants $c_{0}$ and $c_{0}^{\prime}$ such that for all small enough positive $\varepsilon$

$$
B_{\mathbb{H}}\left(\eta\left(z_{0}\right), c_{0} \varepsilon\right) \subset \pi(\mathcal{K}(1-\varepsilon)) \subset B_{\mathbb{H}}\left(\eta\left(z_{0}\right), c_{0}^{\prime} \varepsilon\right) .
$$

Here and hereafter by $B_{\mathbb{H}}(z, \rho)$ we will mean the $\rho$-ball centered at $z$ either in $\mathbb{H}$ (with respect to the hyperbolic metric) or in $\Sigma$ (with respect to the induced quotient metric on $\Sigma)$.

To prove this, we give a much more precise description of our shrinking targets projected to the modular surface $\Sigma$ :

Lemma 4.3. Let $D$ denote the fundamental domain illustrated in diagram (4.5). In the notation of diagram (4.3), for any $r \leq 1$ we have

$$
\pi(\mathcal{K}(r))=\eta\left(\left\{z \in D: \operatorname{Im} z \leq \Delta / r^{2}\right\}\right) .
$$

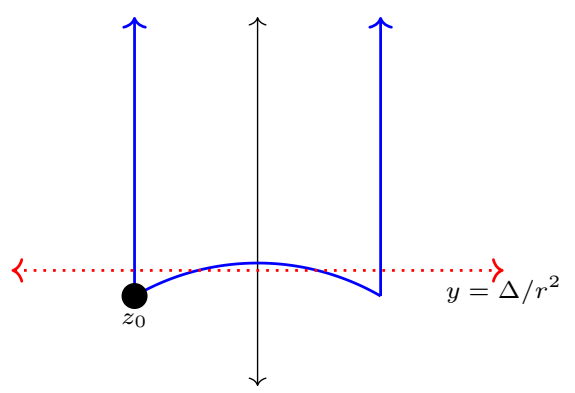

The targets $\pi(\mathcal{K}(r))$ are images of points in the

fundamental domain below the red line.

Proof. We begin by showing that the first set is contained in the second. Choose any lattice $\Lambda \in \mathcal{K}(r)$ and, further, $g \in G$ such that $\Lambda=g \mathbb{Z}^{2}$ and such that $i \cdot g \in D$. It suffices to show $\operatorname{Im}(i \cdot g) \leq \Delta / r^{2}$.

In light of the $\mathrm{SO}(2)$-invariance of the sets $\mathcal{K}(r)$, we may as well assume

$$
g=\left[\begin{array}{cc}
a & b \\
0 & 1 / a
\end{array}\right]
$$


is upper triangular. The fact that $g \mathbb{Z}^{2} \in \mathcal{K}(r)$ implies $a^{2}=\left\|g\left(\begin{array}{l}1 \\ 0\end{array}\right)\right\|^{2} \geq \frac{r^{2}}{\Delta}$, from which we can conclude $\operatorname{Im}(i \cdot g)=\frac{1}{a^{2}} \leq \frac{\Delta}{r^{2}}$.

For the other containment, let $z=i \cdot g \in D$ with $\operatorname{Im} z \leq \Delta / r^{2}$. Again we can assume $g$ is as in (4.12), and we are left with showing that the lattice $\Lambda=g \mathbb{Z}^{2}$ belongs to $\mathcal{K}(r)$. The assumption $i \cdot g=\frac{\frac{1}{a} i-b}{a} \in D$ implies

$$
\left|\frac{b}{a}\right| \leq \frac{1}{2} \text { and } \frac{b^{2}}{a^{2}}+\frac{1}{a^{4}} \geq 1
$$

(the second inequality follows since $z \in D$ implies $|z| \geq 1$ ). We take a non-zero integer vector $\mathbf{v}=\left(\begin{array}{c}m \\ n\end{array}\right)$ and compute its squared norm as

$$
\begin{array}{r}
(m a+n b)^{2}+n^{2} / a^{2}=m^{2} a^{2}+2 m n a b+n^{2}\left(b^{2}+\frac{1}{a^{2}}\right) \\
\geq m^{2} a^{2}+2 m n a b+n^{2} a^{2}=a^{2}\left(m^{2}+2 m n \frac{b}{a}+n^{2}\right) \\
\geq a^{2}\left(m^{2}-2|m n|\left|\frac{b}{a}\right|+n^{2}\right) \geq a^{2}\left(m^{2}-|m n|+n^{2}\right) \geq a^{2} .
\end{array}
$$

In addition, we have $a^{2} \geq r^{2} / \Delta$, since we assumed $\operatorname{Im} z \leq \Delta / r^{2}$, hence $\|\mathbf{v}\| \geq r / \Delta$, which finishes the proof.

Proof of Proposition 4.2. Since the hyperbolic distance on $\mathbb{H}$ satisfies the identity

$$
\sinh \left(\frac{d_{\mathbb{H}}(z, w)}{2}\right)=\frac{|z-w|}{2 \sqrt{\operatorname{Im}(z) \operatorname{Im}(w)}}
$$

we see that the distance from $z_{0}$ to the point $(-1 / 2+x)+i \Delta / r^{2}$ increases as $|x|$ increases. Hence, when $r$ is sufficiently close to 1 so that $\Delta / r^{2}<1$, we get the following estimates for $\pi(\mathcal{K}(r))$ by computing the distance from $z_{0}$ to the point $-1 / 2+i \Delta / r^{2}$ and to the point of intersection of the unit circle with the line $y=\Delta / r^{2}$ :

$$
B_{\mathbb{H}}\left(\eta\left(z_{0}\right),-\ln \left(r^{2}\right)\right) \subset \pi(\mathcal{K}(r)) \subset B_{\mathbb{H}}\left(\eta\left(z_{0}\right), \ln 2+\ln \left(r^{2}-\sqrt{r^{4}-3 / 4}\right)\right) .
$$

Note that

$$
\left.\frac{d\left(-\ln \left(r^{2}\right)\right)}{d r}\right|_{r=1}=\left.\frac{d\left(\ln 2+\ln \left(r^{2}-\sqrt{r^{4}-3 / 4}\right)\right)}{d r}\right|_{r=1}=-2 .
$$

Thus we may choose any $c_{0}<2<c_{0}^{\prime}$ to guarantee (4.10) for small enough positive $\varepsilon$.

\section{A ZERO-ONE LAW ON THE SPACE OF LATTiCES}

We use the following theorem of Maucourant to obtain a zero-one law in the space of lattices.

Theorem 5.1 ([Mau] $)$. Let $\left(B_{\mathbb{H}}\left(p, r_{t}\right)\right)_{t>0}$ be a shrinking family of balls with radius $r_{t}$ in $V$, a finite volume hyperbolic manifold with Liouville measure $\mu$ on its unit tangent bundle $T^{1} V$. Let $\pi$ be the projection from $T^{1} V$ to $V$, and let $\gamma_{t}$ denote the geodesic action of $\mathbb{R}$ on $T^{1} V$. Then for $\mu$-almost every (resp. $\mu$-almost no) $v \in T^{1} V$, the set

$$
\left\{t \geq 0: \pi\left(\gamma_{t} v\right) \in B_{\mathbb{H}}\left(p, r_{t}\right)\right\}
$$


is unbounded (resp. bounded) provided $\int_{0}^{\infty} r_{t} d t$ diverges (resp. converges).

We would like to restate this theorem according to our needs.

Corollary 5.2. Let $\left(B_{\mathbb{H}}\left(\eta\left(z_{0}\right), r_{t}\right)\right)_{t>0}$ be a family of shrinking balls in $\mathbb{H} / \Gamma$ with respect to the quotient metric, with $z_{0}$ as in (4.4). Then for Haar-almost every (resp. almost no) $g \in G / \Gamma$,

$$
\left\{t \geq 0: a_{t} g \in(\pi \circ \phi)^{-1}\left(B_{\mathbb{H}}\left(\eta\left(z_{0}\right), r_{t}\right)\right)\right\}
$$

is unbounded (resp. bounded) provided $\int_{0}^{\infty} r_{t} d t$ diverges (resp. converges).

The difference between Theorem 5.1 and Corollary 5.2 is that our targets $B_{\mathbb{H}}\left(\eta\left(z_{0}\right), r_{t}\right)$ are centered at a branch point of the Riemann surface $\Sigma=\mathbb{H} / \Gamma$, while the proof in [Mau] assumes that the surface $V$ (a finite volume quotient of $\mathbb{H}$ ) admits a fundamental domain that contains a lift of the shrinking targets in its interior. To rectify this difficulty, we let $\Gamma^{\prime} \subset \Gamma$ be a subgroup of finite index with the property that it acts on $\mathbb{H}$ as a fixed-point free group of isometries, or, said in other words, that the image of $\Gamma^{\prime}$ in $\mathrm{PSL}_{2}(\mathbb{R})$ has no torsion ${ }^{7}$. In this case, unlike that of $\Gamma$, the quotient map $\eta: \mathbb{H} \rightarrow \mathbb{H} / \Gamma^{\prime}$ is not only holomorphic, but also has non-zero derivative at each point. This non-degeneracy ensures that $\eta$ is a local diffeomorphism, and that it induces a metric on the quotient $\mathbb{H} / \Gamma^{\prime}$, making it a hyperbolic manifold.

So assuming $\Gamma^{\prime}$ as above, we form a diagram similar to (4.3) with $T^{1} \mathbb{H} / \Gamma^{\prime}$ identified with $T^{1}\left(\mathbb{H} / \Gamma^{\prime}\right)$.

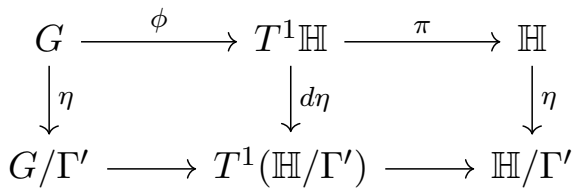

Thus we have a map from the homogeneous space $G / \Gamma^{\prime}$ to the unit tangent bundle of a hyperbolic surface, and this map is a diffeomorphism if $\Gamma^{\prime}$ contains $\pm I$.

Consider the curve $(i, i) \cdot a_{-t / 2}$ in $T^{1} \mathbb{H}$. It gives the velocity vector field over a unitspeed, distance-minimizing curve, that is, the velocity field over a geodesic. Since $g$ acts by isometries, the same is true of $(i, i) \cdot a_{-t / 2} g$ and we have

$$
\phi\left(a_{-t / 2} g\right)=(i, i) \cdot a_{-t / 2} g=\gamma_{t}(\phi(g))
$$

where $\gamma_{t}$ denotes the geodesic flow as in Theorem 5.1. Thus $\phi$ is an $\mathbb{R}$-equivariant map intertwining this diagonal action on $G$ and the geodesic action on $T^{1} \mathbb{H}$. Moreover, since $\eta: \mathbb{H} \rightarrow \mathbb{H} / \Gamma^{\prime}$ preserves the metric, this equivariance is preserved after passing to the map between $G / \Gamma^{\prime}$ and $T^{1}\left(\mathbb{H} / \Gamma^{\prime}\right)$.

Lastly, consider the form $\frac{d x d y d \theta}{y^{2}}$ on $T^{1} \mathbb{H}$. It is invariant under the right action $T^{1} \mathbb{H} \curvearrowleft G$ and so pulls back under $\phi$ to a right invariant top form on $G$. Since $G$ is unimodular, this form is bi-invariant and thus descends to a left-invariant top form on $G / \Gamma^{\prime}$, a Haar measure. On the other hand, $\frac{d x d y d \theta}{y^{2}}$ gives a Liouville measure and by invariance also descends to a top form on $\left(T^{1} \mathbb{H}\right) / \Gamma^{\prime} \simeq T^{1}\left(\mathbb{H} / \Gamma^{\prime}\right)$. By the diagram above we see that this form, pulled back to $G / \Gamma^{\prime}$, is the same Haar measure. We now use a specific torsion-free $\Gamma^{\prime} \subset \Gamma$ to prove Corollary 5.2.

Proof of Corollary 5.2. Set $\Gamma^{\prime}$ in the discussion above to be the congruence subgroup $\Gamma(2) \subset \Gamma$, a torsion-free (up to $\pm I$ ) subgroup of index 6 . As required, $\Gamma^{\prime}$ acts on $\mathbb{H}$ freely as a group of isometries and moreover contains $\pm I$. See Example 5.3 below for an example of one of its fundamental domains to keep in mind for the rest of the proof.

\footnotetext{
${ }^{7}$ We remark that by Selberg's Lemma [Se] any lattice in $G$ has a torsion-free subgroup of finite index.
} 
Combining diagrams (4.3) and (5.3) gives us the following commutative diagram:

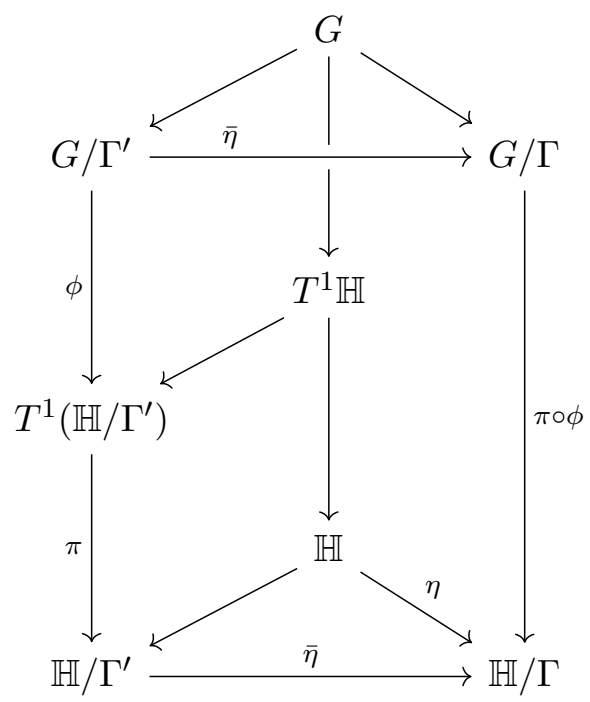

We apologize for the abuse of notation and hope context will remove any ambiguity. We may as well assume $r_{t} \rightarrow 0$, for otherwise ergodicity would prove the result. Note that, even though the map $\bar{\eta}$ has degree 6 , there are only two preimages of $\eta\left(z_{0}\right)$, each with multiplicity 3, see diagram (5.6) below. Thus, in the bottom triangle of diagram (5.5), the preimage of $B_{\mathbb{H}}\left(\eta\left(z_{0}\right), r_{t}\right)$ under $\bar{\eta}$ is the union of two small, disjoint, hyperbolic balls which we write as $\bigcup B_{\mathbb{H}}\left(p_{i}, r_{t}\right)$.

Consider the following subsets of $G / \Gamma$ and $G / \Gamma^{\prime}$ respectively:

$$
\begin{aligned}
T & :=\left\{g \in G / \Gamma: a_{t} g \in(\pi \circ \phi)^{-1} B_{\mathbb{H}}\left(\eta\left(z_{0}\right), r_{t}\right) \text { for an unbounded set of } t>0\right\}, \\
T^{\prime} & :=\left\{g \in G / \Gamma^{\prime}: a_{t} g \in(\pi \circ \phi)^{-1}\left(\bigcup B_{\mathbb{H}}\left(p_{i}, r_{t}\right)\right) \text { for an unbounded set of } t>0\right\} .
\end{aligned}
$$

In the upper triangle of (5.5) one can use the commutativity of the diagram to check that $\bar{\eta}^{-1}(T)=T^{\prime}$. Since the union of two measure zero sets is of measure zero, Theorem 5.1 applies equally well when the targets are a union of two balls (having the same radius for each time $t$ ) in $\mathbb{H} / \Gamma^{\prime}$. Thus we have the required zero-one law for the set $T^{\prime}$. Note that we have actually applied Theorem 5.1 for negative times; cf. (5.4). This is valid since the automorphism of the unit tangent bundle reversing the direction of tangent vectors preserves the Liouville measure. The conclusion now follows since $\bar{\eta}$, being a branched covering map, sends null sets to null sets.

Example 5.3. One fundamental domain for $\Gamma(2)$ can seen as the union of six fundamental domains for $\Gamma$. The preimage $\bar{\eta}^{-1} B_{\mathbb{H}}\left(\eta\left(z_{0}\right), r_{t}\right)$ in $\mathbb{H} / \Gamma(2)$ is the union of two balls. 


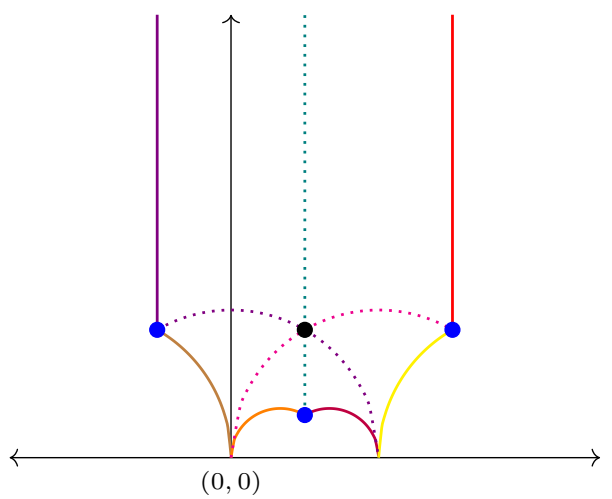

A fundamental domain for $\Gamma(2)$ with one of the balls in its interior. The points at the center of the blue balls represent the same point in $\mathbb{H} / \Gamma(2)$.

We can summarize the results of Sections 4 and 5 as follows:

Theorem 5.4. Let $f$ be any continuous, non-decreasing function $\mathbb{R}_{>0} \rightarrow \mathbb{R}$ with $f(t)<1$. Then the set

$$
T(f):=\left\{\Lambda \in X: a_{t} \Lambda \in \mathcal{K}(f(t)) \text { for an unbounded set of } t>0\right\}
$$

has full (resp. zero) Haar measure if $\int(1-f(t)) d t$ diverges (resp. converges).

Proof. By ergodicity, we may as well assume $f(t)$ converges to 1 as $t \rightarrow \infty$. In this case, Proposition 4.2 and Corollary 5.2 give us the result.

\section{From the sPace of lattices to a submanifold}

We fix the following notation, $w_{y}:=\left[\begin{array}{ll}1 & 0 \\ y & 1\end{array}\right], u_{z}:=\left[\begin{array}{cc}1 & z \\ 0 & 1\end{array}\right]$, and $a_{s}=\left[\begin{array}{cc}e^{s} & 0 \\ 0 & e^{-s}\end{array}\right]$ as before. Observe the relation between the set $T(f)$ in (5.7) and the defining condition in (2.7). If we regard $u$ as a function from $\mathbb{R}$ to $X$ sending $\alpha$ to $\Lambda_{\alpha}=u_{\alpha} \mathbb{Z}^{2}$, we see immediately:

Lemma 6.1. If $\psi$ is as in Theorem 1.6 and $r$ is the function defined by the property $r\left(\frac{1}{2} \ln \frac{t}{\psi(t)}\right)=\sqrt{t \psi(t)}$, which is a special case $m=n=1$ of $(2.6)$, then

$$
D(\psi)^{c}=u^{-1}(T(r)) .
$$

In order to prove Theorem 1.6 using Theorem 5.4, we show that the sets $T(f)$ are invariant, in some sense, under the action of $w_{y}$ and $a_{s}$. This allows us to conclude that the Haar measure of $T(f)$ is locally controlled by the Lebesgue measure of $u^{-1}(T(f))$. The argument is similar to that of [Da] (cf. Proposition 3.2). The effect of perturbing a lattice by $w_{y}$ or $a_{s}$ will be computed in terms of the function

$$
\delta: \Lambda \mapsto \sqrt{\Delta} \cdot \inf _{\mathbf{x} \in \Lambda \backslash\{0\}}\|\mathbf{x}\|
$$

as in (2.10). With the help of this function, the sets $T(f)$ can be rewritten as

$$
T(f)=\left\{\Lambda \in X: a_{s} \Lambda \in \delta^{-1}[f(s), 1] \text { for an unbounded set of } s\right\} .
$$

Proposition 6.2. We have

$$
\delta\left(a_{s} w_{y} \Lambda\right) \leq\left(1+|y| e^{-2 s}\right) \delta\left(a_{s} \Lambda\right) \quad \text { and } \quad \delta\left(a_{s} \Lambda\right) \leq\left(1+|y| e^{-2 s}\right) \delta\left(a_{s} w_{y} \Lambda\right) .
$$


Proof. Note the commutation relations,

$$
a_{s} w_{y}=a_{s} w_{y} a_{-s} a_{s}=w_{y e^{-2 s}} a_{s} .
$$

Now let $\mathbf{v} \in \mathbb{R}^{2}$ be a vector in the lattice $\Lambda$ such that $\delta\left(a_{s} \Lambda\right)=\sqrt{\Delta} \cdot\left\|a_{s} \mathbf{v}\right\|$. We compute

$$
\begin{aligned}
\left\|a_{s} w_{y} \mathbf{v}\right\|-\left\|a_{s} \mathbf{v}\right\| & =\left\|w_{y e^{-2 s}} a_{s} \mathbf{v}\right\|-\left\|a_{s} \mathbf{v}\right\| \\
& \leq\left\|w_{y e^{-2 s}}-I\right\|\left\|a_{s} \mathbf{v}\right\| \\
& \leq|y| e^{-2 s}\left\|a_{s} \mathbf{v}\right\| .
\end{aligned}
$$

This gives

$$
\sqrt{\Delta} \cdot\left\|a_{s} w_{y} v\right\| \leq\left(1+|y| e^{-2 s}\right) \delta\left(a_{s} \Lambda\right)
$$

which implies the first inequality in (6.2). The other estimate follows similarly.

Theorem 6.3. For a continuous, non-decreasing function $f: \mathbb{R}_{>0} \rightarrow \mathbb{R}$ with $f(t)<1$, the set $u^{-1}(T(f))$ has full (resp. zero) Lebesgue measure provided $\int(1-f(t)) d t$ diverges (resp. converges).

Proof. Choose any $z_{1} \in \mathbb{R}$. There exists an $\varepsilon>0$ such that the map

$$
\Phi: W:=(-\varepsilon, \varepsilon)^{2} \times\left(z_{1}-\varepsilon, z_{1}+\varepsilon\right) \rightarrow X
$$

sending $(y, s, z)$ to the lattice generated by $w_{y} a_{s} u_{z}$ is a diffeomorphism. Depending on the convergence of the integral in question, we will show that $u^{-1}(T(f)) \cap\left(z_{1}-\varepsilon, z_{1}+\varepsilon\right)$ has full or zero measure. This clearly suffices to prove the theorem.

Proof of the convergence case. Assume $\int(1-f)$ converges. Define

$$
h(t):=\left(\tau_{-\varepsilon} f\right)(t) \cdot\left(1-\varepsilon e^{-2 t}\right)
$$

where $\tau$ is translation, defined by

$$
\left(\tau_{-\varepsilon} f\right)(t):=f(t-\varepsilon)
$$

The function $h$ is still non-decreasing, continuous, and bounded from above by 1 , and the set $T(h)$ still is well defined even though $h(t)$ is only defined for $t>\varepsilon$. Our integrability assumption on $f$ and Theorem 5.4 imply that $T(h)$ has zero measure.

Claim 6.3.1. Let $|y|,|s|<\varepsilon$. If $\Lambda \in T(f)$, then $w_{y} a_{s} \Lambda \in T(h)$.

Proof. Let $\Lambda \in T(f)$ and $|s|<\varepsilon$. Let $\left(t_{n}\right)$ be a sequence witnessing $\Lambda \in T(f)$. Then

$$
\delta\left(a_{t_{n}-s} a_{s} \Lambda\right)=\delta\left(a_{t_{n}} \Lambda\right) \geq f\left(t_{n}\right)=\left(\tau_{s} f\right)\left(t_{n}-s\right) \geq\left(\tau_{-\varepsilon} f\right)\left(t_{n}-s\right) .
$$

So $a_{s} \Lambda \in T\left(\tau_{-\varepsilon} f\right)$.

Now say $\Lambda \in T\left(\tau_{-\varepsilon} f\right)$ and let $|y|<\varepsilon$. Let $t_{n}$ be a sequence witnessing $\Lambda \in T\left(\tau_{-\varepsilon} f\right)$. Using Proposition 6.2 we see that

$$
\delta\left(a_{t_{n}} w_{y} \Lambda\right) \geq \frac{\delta\left(a_{t_{n}} \Lambda\right)}{1+\varepsilon e^{-2 t_{n}}} \geq\left(\tau_{-\varepsilon} f\right)\left(t_{n}\right)\left(1-\varepsilon e^{-2 t_{n}}\right) .
$$

Hence $w_{y} \Lambda \in T(h)$, and the claim is proved.

An application of the above claim to $\Lambda=\Lambda_{z}$ shows that $\Phi$ maps the set

$$
(-\varepsilon, \varepsilon)^{2} \times\left(u^{-1}(T(f)) \cap\left(z_{1}-\varepsilon, z_{1}+\varepsilon\right)\right)
$$

to a set of measure zero. The Fubini Theorem and the local equivalence of Haar measure and Lebesgue measure shows that $u^{-1}(T(f)) \cap\left(z_{1}-\varepsilon, z_{1}+\varepsilon\right)$ has Lebesgue measure zero.

Proof of the divergence case. The strategy is similar: we show that, in terms of the local coordinates, the union of planes above $u^{-1}(T(f))$ contains some full measure set. As before, this amounts to finding some appropriate function $h$ such that $T(h)$ is full measure 
and such that the family of planes contains $T(h)$ as a subset. A naive guess based on Proposition 6.2 would be to use the function $f(\cdot)\left(1+\varepsilon e^{-2(\cdot)}\right)$. However this function is not monotonic; indeed it can even be greater than 1 on certain intervals depending on how pathological $f$ is. The adjustment we make below is to choose $h$ more carefully and then to throw out some measure 0 set to ensure it is contained in our family of planes.

Let $\int(1-f)$ diverge. Then $\int \frac{1-f}{2}$ diverges too. The function $\frac{1+f}{2}$ certainly satisfies the hypotheses of Theorem 5.4, hence $T\left(\frac{1+f}{2}\right)$ has full measure. The same conclusion holds if we replace $f$ by $\tau_{\varepsilon} f$. Another application of Theorem 5.4 shows that $T\left(1-\varepsilon e^{-2(\cdot)}\right)$ has zero measure.

Claim 6.3.2. Let $|y|,|s|<\varepsilon$. Then $a_{s} w_{y}\left(T\left(\frac{1+\tau_{\varepsilon} f}{2}\right) \backslash T\left(1-\varepsilon e^{-2(\cdot)}\right)\right) \subset T(f)$.

Proof. Take

$$
\Lambda \in T\left(\frac{1+\tau_{\varepsilon} f}{2}\right) \backslash T\left(1-\varepsilon e^{-2(\cdot)}\right)
$$

and let $t_{n}$ be a sequence witnessing $\Lambda \in T\left(\frac{1+\tau_{\varepsilon} f}{2}\right)$. We can assume that the sequence satisfies

$$
\frac{1+\left(\tau_{\varepsilon} f\right)\left(t_{n}\right)}{2} \leq \delta\left(a_{t_{n}} \Lambda\right)<1-\varepsilon e^{-2 t_{n}}
$$

Since $\min (a, b) \leq \frac{a+b}{2}$, we have

$$
\min \left(\left(\tau_{\varepsilon} f\right)(t)+\varepsilon e^{-2 t}, 1-\varepsilon e^{-2 t}\right) \leq \frac{1+\left(\tau_{\varepsilon} f\right)(t)}{2} .
$$

Inequalities (6.3) and (6.4) applied to our sequence show that

$$
\left(\tau_{\varepsilon} f\right)\left(t_{n}\right)+\varepsilon e^{-2 t_{n}} \leq \delta\left(a_{t_{n}} \Lambda\right)
$$

Now we estimate, using Proposition 6.2 and the previous inequality:

$$
\begin{aligned}
& \delta\left(a_{t_{n}} w_{y} \Lambda\right) \geq \frac{\delta\left(a_{t_{n}} \Lambda\right)}{1+\varepsilon e^{-2 t_{n}}} \geq \frac{\left(\tau_{\varepsilon} f\right)\left(t_{n}\right)+\varepsilon e^{-2 t_{n}}}{1+\varepsilon e^{-2 t_{n}}} \\
& >\frac{\left(\tau_{\varepsilon} f\right)\left(t_{n}\right)\left(1+\varepsilon e^{-2 t_{n}}\right)}{1+\varepsilon e^{-2 t_{n}}}=\left(\tau_{\varepsilon} f\right)\left(t_{n}\right) .
\end{aligned}
$$

Hence $w_{y} \Lambda \in T\left(\tau_{\varepsilon} f\right)$. And the argument in the convergence part shows that

$$
a_{s} w_{y} \Lambda \in T\left(\tau_{-\varepsilon}\left(\tau_{\varepsilon} f\right)\right)=T(f),
$$

and our claim is proved.

We use this claim to show that

$$
\Phi(W) \cap\left(T\left(\frac{1+\tau_{\varepsilon} f}{2}\right) \backslash T\left(1-\varepsilon e^{-2(\cdot)}\right)\right),
$$

a set of full measure with respect to the chart, is contained in

$$
\Phi\left((-\varepsilon, \varepsilon)^{2} \times\left(u^{-1}(T(f)) \cap\left(z_{1}-\varepsilon, z_{1}+\varepsilon\right)\right)\right)
$$

as follows: take any $\Lambda$ in the first set. Since it is in the chart, we may write $\Lambda=w_{y} a_{s} \Lambda_{z}$ or equivalently, $a_{-s} w_{-y} \Lambda=\Lambda_{z}$. Claim 6.3.2 then gives us exactly what we need.

Again, by Fubini and the local equivalence of Lebesgue and Haar measure, we see that the set

$$
u^{-1}(T(f)) \cap\left(z_{1}-\varepsilon, z_{1}+\varepsilon\right)
$$

has full Lebesgue measure, and the divergence case is proved. 
We now specialize to the case where $f$ is the function $r$ in Lemma 6.1 to give a proof of Theorem 1.6:

Corollary 6.4. Let $\psi$ be a continuous, non-increasing function such that $t \psi(t)$ is nondecreasing and $t \psi(t)<1$ for sufficiently large $t$. Then the Lebesgue measure of $D(\psi)$ (resp. of $\left.D(\psi)^{c}\right)$ is zero if

$$
\sum_{k}\left(\psi_{1}(k)-\psi(k)\right)=\sum_{k}\left(\frac{1}{k}-\psi(k)\right)=\infty \quad(\text { resp. }<\infty) .
$$

Proof. Applying Theorem 6.3, we see that $D(\psi)^{c}=u^{-1}(T(r))\left(\operatorname{resp} . D(\psi)=u^{-1}(T(r))^{c}\right)$ has measure zero if the integral

$$
\int(1-r(s)) d s
$$

converges (resp. diverges). It remains to show that the convergence of (6.6) is equivalent to the convergence of (6.5). Using the definition of $r$ (cf. (4.8)), we compute

$$
\begin{aligned}
\int(1-r(s)) d s & =\int(1-\sqrt{t \psi(t)}) d\left(\frac{1}{2} \ln \frac{t}{\psi(t)}\right) \\
& =\int(1-\sqrt{t \psi(t)}) d\left(\ln \frac{t}{\sqrt{t \psi(t)}}\right) \\
& =\int(1-\sqrt{t \psi(t)}) d(\ln t-\ln \sqrt{t \psi(t)}) .
\end{aligned}
$$

Note that the integrals coming after the first equality are taken in the Riemann-Stieltjes sense.

Observe that $\int(1-\sqrt{t \psi(t)}) d(\ln \sqrt{t \psi(t)})$ is finite, and that the ratio

$$
\frac{1-t \psi(t)}{1-\sqrt{t \psi(t)}}=1+\sqrt{t \psi(t)}
$$

is bounded between two positive constants. Thus the convergence of (6.6) is equivalent to that of $\int\left(\frac{1}{t}-\psi(t)\right) d t$, which, in view of the monotonicity of $\psi$, is in turn equivalent to the convergence of the sum in (6.5).

\section{Appendix: Proof of Theorem 3.11}

As promised, this section will verify Condition (3.7) for norms in $\mathbb{R}^{2}$ whose norm balls are not parallelograms. Recall that $G$ and $\Gamma$ denote the groups $\mathrm{SL}_{2}(\mathbb{R})$ and $\mathrm{SL}_{2}(\mathbb{Z})$ respectively, $X$ denotes the space of unimodular lattices $G / \Gamma$, and $F, H \subset G$ are as in (3.10). Also recall that when $Z \subset X$ is a one-dimensional submanifold and with $F, H$ as above, the $(F, H)$ transversality of $Z$ is equivalent to (3.11).

Recall that the critical locus $\mathcal{K}_{\nu}(1)$ is, by definition of $\Delta_{\nu}$, the set of lattices of smallest covolume (necessarily 1 ) intersecting the symmetric convex domain $B_{\nu}\left(1 / \sqrt{\Delta_{\nu}}\right)$ trivially. The existence of the one-dimensional submanifold containing the critical set follows from the work of Mahler whose results we make free use of. Since his work is phrased in terms of convex domains, and since our parameterization of the hypothesized one-dimensional submanifold $Z$ comes from the boundary of such a domain, we switch from the language of norms to that of bounded symmetric convex domains in $\mathbb{R}^{2}$.

Given such a domain $B \subset \mathbb{R}^{2}$, a lattice $\Lambda$ is called $B$-admissible if it intersects $B$ trivially. A lattice $\Lambda$ is called $B$-critical if it is of smallest covolume amongst all admissible lattices. The critical determinant $\Delta_{B}$ associated to $B$ is defined to be this smallest covolume attained. Note that by Minkowski's convex body theorem [Ca2, Theorem II, §III.2.2] it is 
necessarily positive, and, moreover, $\Delta_{B} \geq \frac{\operatorname{area}(B)}{4}$, with equality if $B$ is a parallelogram. The set of all $B$-critical lattices will be called the critical locus of $B$.

As for our previous notation, $\Delta_{\nu}$ is nothing but the critical number of the convex domain $B_{\nu}(1)$, and $\mathcal{K}_{\nu}(1)$ is the set of $B_{\nu}\left(1 / \sqrt{\Delta_{\nu}}\right)$-critical lattices. The following theorem (from $[\mathrm{Ca} 2, \S \mathrm{V} .8 .3])$ is of fundamental importance.

Theorem 7.1. Let $\Lambda$ be B-critical, and let $C$ be the boundary of $B$. Then one can find three pairs of points $\pm \mathbf{p}, \pm \mathbf{q}, \pm \mathbf{r}$ of the lattice on $C$. Moreover these three points can be chosen such that

$$
\mathbf{p}=\mathbf{q}-\mathbf{r}
$$

and any two vectors among $\mathbf{p}, \mathbf{q}, \mathbf{r}$ form a basis of $\Lambda$.

Conversely, if $\mathbf{p}, \mathbf{q}, \mathbf{r}$ satisfying (7.1) are on $C$, then the lattice generated by $\mathbf{p}$ and $\mathbf{q}$ is $B$-admissible. Furthermore no additional (excluding the six above) point of $\Lambda$ is on $C$ unless $B$ is a parallelogram.

This theorem shows that candidates for critical lattices may be found by tracing along the boundary of $B$ and finding two other points satisfying equation (7.1). However such a configuration of points does not necessarily yield a critical lattice. Hexagons, for example, have only one critical lattice (see Lemma 13 in [Ca2, V.8.3]). One even has domains for which the critical locus is a fractal set, see [KRS] for a discussion of this topic and concrete examples. However, the following notion due to Mahler gives a class of domains whose critical loci are well behaved.

Definition 7.2. A convex symmetric bounded domain $B$ in $\mathbb{R}^{2}$ is said to be irreducible if each convex symmetric domain $B^{\prime} \varsubsetneqq B$ has $\Delta_{B^{\prime}}<\Delta_{B}$. We say $B$ is reducible if it is not irreducible, that is, if there exists $\underset{B^{\prime}}{\varsubsetneqq} B$ with $\Delta_{B^{\prime}}=\Delta_{B}$.

The following was proved by Mahler in the 1940s:

Lemma 7.3 ([Ma3], Lemmata 5 and 9). Assume $B$ is not a parallelogram and is irreducible. Then:

(i) for each $\mathbf{p} \in \partial B$ there is exactly one $B$-critical lattice containing $\mathbf{p}$;

(ii) for each $B$-critical lattice $\Lambda$ and each $\mathbf{q}, \mathbf{r} \in \partial B \cap \Lambda$, all points of the line segment between $\mathbf{q}, \mathbf{r}$ different from $\mathbf{q}, \mathbf{r}$ are interior points of $B$.

Lemma 7.4 ([Ma4], Theorem 3). If B is not a parallelogram and is irreducible, the boundary $\partial B$ is a $\mathcal{C}^{1}$-submanifold of $\mathbb{R}^{2}$.

Capitalizing on these results, we have the following regularity statement:

Corollary 7.5. Suppose $B$ is not a parallelogram and is irreducible. Assume further that $B$ is scaled so that $\Delta_{B}=1$. Then the locus of $B$-critical lattices is a $\mathcal{C}^{1}$-submanifold of $X$.

Proof. Let $C$ denote the boundary of $B$. In light of Lemma 7.4 we have a local diffeomorphism from $\mathbb{R}$ to $C$ given by $t \mapsto \mathbf{p}(t)=(a(t), b(t))$. Fix a point, say $\mathbf{p}:=\mathbf{p}\left(t_{0}\right)$, and consider

$$
\{\mathbf{v}: \operatorname{det}(\mathbf{p}, \mathbf{v})=1\} \cap C
$$

By Lemma 7.3 (i) we know that there is a unique critical lattice containing $\mathbf{p}$ so that, by Theorem 7.1, the above intersection consists of two points. Let $\mathbf{q}=\mathbf{p}\left(t_{1}\right)$ denote one of 
those points.

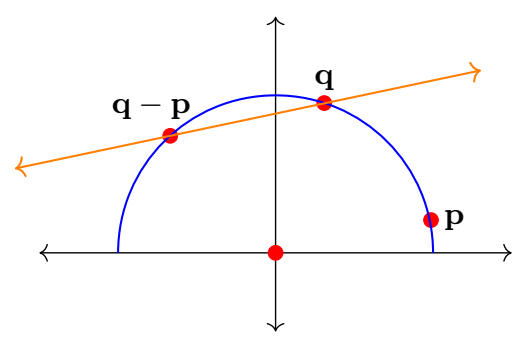

The intersection of $C$ and the line $\operatorname{det}(\mathbf{p}, \cdot)=1$ consists of two points.

We claim that there is a neighborhood $W$ of $\mathbf{q}$ such that for points $\mathbf{p}(t)$ sufficiently close to $\mathbf{p}$, the intersection

$$
\{\mathbf{v}: \operatorname{det}(\mathbf{v}, \mathbf{p}(t))=1\} \cap W \cap C
$$

will consist of a unique point $\mathbf{q}(t)$. Moreover, this assignment is $\mathcal{C}^{1}$-differentiable. The corollary clearly follows from this claim.

To prove the claim, we make a straightforward appeal to the implicit function theorem. First note that since $C$ is a $\mathcal{C}^{1}$-submanifold, the local immersion theorem (see [Ru, Theorem 9.32]) guarantees a neighborhood $\mathbf{q} \in W$ and the existence of a non-degenerate, $\mathcal{C}^{1}$-differentiable function $f: W \rightarrow \mathbb{R}$ such that $C \cap W=f^{-1}(0)$. Then consider the following function defined in a neighborhood of $\left(\mathbf{q}, t_{0}\right) \in \mathbb{R}^{3}$ :

$$
F(x, y, t):=(a(t) y-b(t) x-1, f(x, y)) .
$$

Clearly $F\left(\mathbf{q}, t_{0}\right)=0$. Moreover we have that the derivative of $F$ at $\left(\mathbf{q}, t_{0}\right)$ is given by the matrix

$$
\left[\begin{array}{ccc}
-b\left(t_{0}\right) & a\left(t_{0}\right) & a^{\prime}\left(t_{0}\right) b\left(t_{1}\right)-b^{\prime}\left(t_{0}\right) a\left(t_{1}\right) \\
\frac{\partial f}{\partial x}(\mathbf{q}) & \frac{\partial f}{\partial y}(\mathbf{q}) & 0
\end{array}\right] .
$$

In view of Lemma 7.3(ii), the tangent line to $C$ at $\mathbf{q}$ cannot be parallel to $\mathbf{p}$, or, equivalently, the gradient of $f$ at $\mathbf{q}$ cannot be perpendicular to $\mathbf{p}$. Hence the leftmost $2 \times 2$ minor of the matrix (7.3) is non-zero, and we can apply the implicit function theorem (see $\left[\mathrm{Ru}\right.$, Theorem 9.28]) to get a $\mathcal{C}^{1}$-function $\mathbf{q}(t)=(c(t), d(t))$ defined for points $t$ near $t_{0}$ and mapping to a neighborhood of $\mathbf{q}$ such that locally,

$$
F(x, y, t)=(0,0) \text { if and only if }(x, y)=\mathbf{q}(t) .
$$

This proves the claim. The function $[\mathbf{p}(t), \mathbf{q}(t)]$ descends to a local parameterization of the critical locus.

Proof of Theorem 3.11. Let $B$ be the $\nu$-ball of radius $1 / \sqrt{\Delta_{\nu}}$. In particular, we have $\Delta_{B}=1$, so that the critical locus is a subset of $X$. As before, let $C$ denote the boundary of $B$. We first treat the case when $B$ is irreducible. Since the problem is local, we fix a critical lattice $\Lambda$ and check the transversal condition at $\Lambda$.

Fix a point $\mathbf{p}=r_{t_{0}}\left(\cos t_{0}, \sin t_{0}\right) \in \Lambda \cap C$ with $r_{t_{0}}>0$. In general every point on $C$ can be written as $\mathbf{p}(t):=r_{t}(\cos t, \sin t)$ with $r_{t}>0$, but Lemma 7.4 and convexity allow us to go further and say that this local parameterization of $C$ is actually continuously differentiable. Applying Corollary 7.5 we have a local parametrization of the critical locus in the form $M(t) \mathbb{Z}^{2}$, where $M(t):=[\mathbf{p}(t), \mathbf{r}(t)]$ with $M\left(t_{0}\right) \mathbb{Z}^{2}=\Lambda$.

Moreover, we can assume that $\operatorname{det}(\mathbf{p}(t), \mathbf{r}(t))=1$, that $\mathbf{p}(t)+\mathbf{r}(t) \in C$, and that $\mathbf{p}\left(t_{0}\right)$ has the smallest angle (equal to $t_{0}$ ) modulo $[0,2 \pi)$ among vectors in $\Lambda \cap C$ (see diagram (7.5) below). We write out the coordinates of $\mathbf{p}$ and $\mathbf{r}$ as

$$
M(t)=\left[\begin{array}{ll}
a(t) & c(t) \\
b(t) & d(t)
\end{array}\right]
$$


Our goal is to show that the differential of the curve $M(t) \mathbb{Z}^{2}$ at $t_{0}$, which is identified with an element of $\mathfrak{s l}_{2}(\mathbb{R})$, is not contained in the Lie algebra $\operatorname{Lie}(P) \subset \mathfrak{s l}_{2}(\mathbb{R})$ of uppertriangular matrices. Under the map $g \mapsto g \Lambda$, this curve of lattices is the image of a curve in $G$ passing through the identity, namely $M(t) M\left(t_{0}\right)^{-1}$. Writing $M^{\prime}\left(t_{0}\right)$ for the componentwise derivative of $M(t)$ at $t_{0}$, we see that we are left with showing that the bottom left entry of

$$
M^{\prime}\left(t_{0}\right) M\left(t_{0}\right)^{-1}=\left[\begin{array}{ll}
a^{\prime}\left(t_{0}\right) & c^{\prime}\left(t_{0}\right) \\
b^{\prime}\left(t_{0}\right) & d^{\prime}\left(t_{0}\right)
\end{array}\right]\left[\begin{array}{cc}
d\left(t_{0}\right) & -c\left(t_{0}\right) \\
-b\left(t_{0}\right) & a\left(t_{0}\right)
\end{array}\right]
$$

is never zero. For the sake of contradiction assume it is zero. This can happen if and only if

$$
\left(b^{\prime}\left(t_{0}\right), d^{\prime}\left(t_{0}\right)\right)=\lambda\left(b\left(t_{0}\right), d\left(t_{0}\right)\right) \text { for some } \lambda \in \mathbb{R} .
$$

We derive a contradiction by noting the following claims:

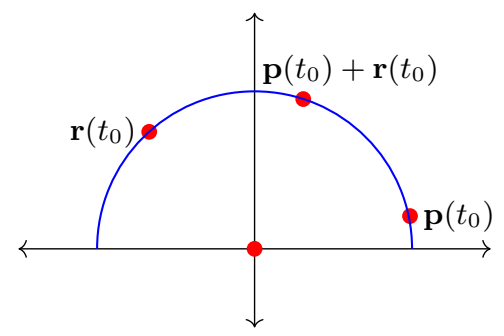

$b^{\prime}\left(t_{0}\right)$ and $d^{\prime}\left(t_{0}\right)$ must have different signs.

On the other hand $b\left(t_{0}\right)$ and $d\left(t_{0}\right)$ are positive.

Claim 7.5.1. $b\left(t_{0}\right)$ and $d\left(t_{0}\right)$ are strictly positive.

Proof. First, note that $d\left(t_{0}\right)>0$ by choice on minimality of the angle of $\mathbf{p}\left(t_{0}\right)$ and by the symmetry of $B$. Moreover, (7.4) certainly cannot hold if $b\left(t_{0}\right)=0$ since that would also imply $b^{\prime}\left(t_{0}\right)=0$. Both of these cannot vanish simultaneously since $B$ is convex, and the origin is its interior point.

Claim 7.5.2. $b^{\prime}\left(t_{0}\right)>0$ and $d^{\prime}\left(t_{0}\right)<0$.

Proof. We first show that $b^{\prime}\left(t_{0}\right)>0$. If not, then the slope of the tangent line to $C$ at $\mathbf{p}\left(t_{0}\right)$ is non-negative; hence, by the convexity of $B$, the curve

$$
\left\{\mathbf{p}(t): t_{0} \leq t \leq \pi\right\}
$$

is contained in the half-plane $\left\{(x, y): y \leq b\left(t_{0}\right)\right\}$. On the other hand, the point $\mathbf{p}\left(t_{0}\right)+\mathbf{r}\left(t_{0}\right)$ lies on the curve (7.6), and its $y$-coordinate is equal to $b\left(t_{0}\right)+d\left(t_{0}\right)$, which is strictly larger that $b\left(t_{0}\right)$ by Claim 7.5.1, a contradiction.

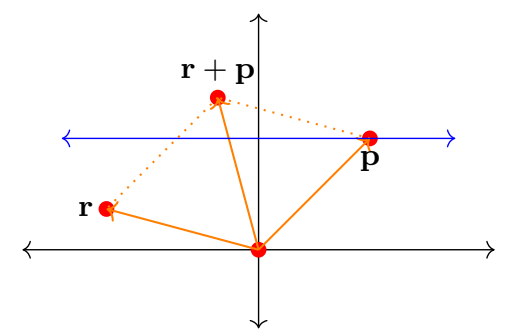

$b^{\prime}\left(t_{0}\right)$ cannot be less than or equal to 0.

The inequality $d^{\prime}\left(t_{0}\right)<0$ is proved by the above argument applied to the tangent line to $C$ at $\mathbf{r}\left(t_{0}\right)$. 
Now the above two claims and condition (7.4) are incompatible, and we have reached our contradiction. This shows that the critical locus of $B$ is transversal to the distribution generated by $\operatorname{Lie}(P)$ as required.

In order to generalize to the case when $B$ is reducible, we use Mahler's important result that each convex, bounded, symmetric domain $B$ contains an irreducible $B^{\prime}$ which has the same critical determinant, that is $\Delta_{B}=\Delta_{B^{\prime}}$ (see [Ma3, Theorem 1]). We claim that since $B$ is not a parallelogram, this irreducible $B^{\prime}$ cannot be a parallelogram either. For in this case

$$
\Delta_{B^{\prime}}=\frac{\operatorname{area}\left(B^{\prime}\right)}{4}<\frac{\operatorname{area}(B)}{4} \leq \Delta_{B}
$$

which is a contradiction.

Finally, the containment $B^{\prime} \subset B$ and the equality $\Delta_{B^{\prime}}=\Delta_{B}$ show that the $B$-critical locus is contained in the $B^{\prime}$-critical locus. This completes the proof.

\section{REFERENCES}

[AD] N. Andersen and W. Duke, On a theorem of Davenport and Schmidt, preprint (2019), https://arxiv.org/abs/1905.05236.

[AGK] J. An, L. Guan and D. Kleinbock, Nondense orbits on homogeneous spaces and applications to geometry and number theory, preprint (2020), https://arxiv.org/pdf/2001.05174.pdf.

[BFKRW] R. Broderick, L. Fishman, D. Kleinbock, A. Reich and B. Weiss, The set of badly approximable vectors is strongly $\mathcal{C}^{1}$ incompressible, Math. Proc. Cambridge Philos. Soc. 153 (2012), no. 2, 319-339.

[BFS] R. Broderick, L. Fishman and D. Simmons, Badly approximable systems of affine forms and incompressibility on fractals, J. Number Theory 133, no. 7 (2013), 2186-2205.

[Ca1] J.W.S. Cassels, An Introduction to Diophantine Approximation, Cambridge Tracts in Mathematics and Physics, Cambridge University Press, London, 1957.

[Ca2] - An introduction to the geometry of numbers, Die Grundlehren der mathematischen Wissenschaften, Band 99, Springer-Verlag, Berlin-New York, 1971.

[CGGMS] S. Chow, A. Ghosh, L. Guan, A. Marnat and D. Simmons, Diophantine transference inequalities: weighted, inhomogeneous, and intermediate exponents, Ann. Sc. Norm. Super. Pisa Cl. Sci. (5), to appear.

[Da] S. G. Dani, Divergent trajectories of flows on homogeneous spaces and Diophantine approximation, J. Reine Angew. Math. 359 (1985), 55в ГY 89.

[DS1] H. Davenport and W. M. Schmidt, Dirichlet's theorem on diophantine approximation, in: Symposia Mathematica, Vol. IV (INDAM, Rome, 1968/69), 1970.

[DS2] , Dirichlet's theorem on diophantine approximation. II, Acta Arith. 16 (1969/1970) 413-424.

[EW] M. Einsiedler and T. Ward, Diophantine Problems and Homogeneous Dynamics, in: Dynamics and analytic number theory, pp. 258-288, Cambridge University Press, London, 2016.

[F] Fuchs, L., Abelian groups, International Series of Monographs on Pure and Applied Mathematics, Pergamon Press, New York-Oxford-London-Paris, 1960.

[GGM] N. M. Glazunov, A. S. Golovanov and A. V. Malyshev, Proof of the Minkowski conjecture on the critical determinant of the region $|x|^{p}+|y|^{p}<1$, Zap. Nauchn. Sem. Leningrad. Otdel. Mat. Inst. Steklov (LOMI) 151 (1986), 40-53.

[J] V. Jarník, Zur metrischen theorie der diophantischen approximationen, Prace mat-fiz. 36, no. 1 (1928), 91-106.

[Kl] D. Kleinbock, Nondense orbits of flows on homogeneous spaces, Ergodic Theory Dynam. Systems 18 (1998), 373-396.

[KM] D. Kleinbock and G. A. Margulis, Logarithm laws for flows on homogeneous spaces, Invent. Math. 138 (1999), no. 3, 451-494. 
[KRS] D. Kleinbock, A. Rao, S. Sathiamurthy, Critical loci of convex domains in the plane, preprint (2020), https://arxiv.org/pdf/2003.13829.pdf.

[KWa] D. Kleinbock and N. Wadleigh, A zero-one law for improvements to Dirichlet's Theorem, Proc. Amer. Math. Soc. 146 (2018), no. 5, 1833-1844.

[KWe] D. Kleinbock and B. Weiss, Values of binary quadratic forms at integer points and Schmidt games, in: Recent trends in ergodic theory and dynamical systems (Vadodara, 2012), pp. 77-92, Contemp. Math., vol. 631, Amer. Math. Soc., Providence, RI, 2015.

[KZ] A. Korkine and G. Zolotareff, Sur les formes quadratiques positives, Math. Ann. 11 (1877), 242-292.

[Ma1] K. Mahler, On lattice points in n-dimensional star bodies. I. Existence theorems, Proc. Roy. Soc. London. Ser. A. 187 (1946), 151-187.

[Ma2] - On lattice points in $n$ dimensional star bodies. II. Reducibility theorems, Nederl. Akad. Wetensch., Proc. 49 (1946), 444-454.

[Ma3] $\quad$ On irreducible convex domains, Nederl. Akad. Wetensch., Proc. 50 (1947), 98-107.

[Ma4] - On the minimum determinant and the circumscribed hexagons of a convex domain, Nederl. Akad. Wetensch., Proc. 50 (1947), 326-337.

[Mar] J. Martinet, Perfect lattices in Euclidean spaces, Grundlehren der Mathematischen Wissenschaften, Band 327, Springer-Verlag, Berlin, 2003.

[Mau] F. Maucourant, Dynamical Borel-Cantelli lemma for hyperbolic spaces. Israel J. Math. 152 (2006), 143-155.

[Mc] C. McMullen, Winning sets, quasiconformal maps and Diophantine approximation, Geom. Funct. Anal. 20 (2010), no. 3, 726-740.

[Mo] S. Morimoto, Zur Theorie der Approximation einer irrationalen Zahl durch rationale Zahlen, Tohoku Math. J. 45 (1939), 177-187.

[Ru $\quad$ W. Rudin, Principles of mathematical analysis, International Series in Pure and Applied Mathematics, McGraw-Hill Book Co., New York-Auckland-Düsseldorf, 1976.

[S1] W. M. Schmidt, On badly approximable numbers and certain games, Trans. Amer. Math. Soc. 123 (1966), 178-199.

[S2] _ Badly approximable systems of linear forms, J. Number Theory 1 (1969), 139154.

[S3] - Diophantine approximation and certain sequences of lattices, Acta Arith. 18 (1971), no. 1, 165-178.

[S4] Diophantine approximation, Lecture Notes in Mathematics, vol. 785, SpringerVerlag, Berlin, 1980.

[Se] A. Selberg, On discontinuous groups in higher-dimensional symmetric spaces, in: Contributions to Function Theory, pp. 147-164, Tata Institute of Fundamental Research, Bombay, 1960.

[W] M. Waldschmidt, Recent advances in Diophantine approximation, in: Number theory, analysis and geometry (Lang memorial volume), pp. 659-704, Springer, New York, 2012.

Brandeis University, WALtham MA 02454-9110 kleinboc@brandeis.edu

Brandeis University, Waltham MA 02454-9110 anrg@brandeis.edu 